\title{
Investigating the collision energy dependence of $\eta / s$ in the beam energy scan at the BNL Relativistic Heavy Ion Collider using Bayesian statistics
}

\author{
Jussi Auvinen, ${ }^{*}$ Jonah E. Bernhard, and Steffen A. Bass \\ Department of Physics, Duke University, Durham, North Carolina 27708, USA \\ Iurii Karpenko \\ INFN - Sezione di Firenze, I-50019 Sesto Fiorentino, Firenze, Italy
}

(Received 12 June 2017; revised manuscript received 8 December 2017; published 13 April 2018)

\begin{abstract}
We determine the probability distributions of the shear viscosity over the entropy density ratio $\eta / s$ in the quark-gluon plasma formed in $\mathrm{Au}+\mathrm{Au}$ collisions at $\sqrt{s_{N N}}=19.6,39$, and $62.4 \mathrm{GeV}$, using Bayesian inference and Gaussian process emulators for a model-to-data statistical analysis that probes the full input parameter space of a transport + viscous hydrodynamics hybrid model. We find the most likely value of $\eta / s$ to be larger at smaller $\sqrt{s_{N N}}$, although the uncertainties still allow for a constant value between 0.10 and 0.15 for the investigated collision energy range.
\end{abstract}

DOI: 10.1103/PhysRevC.97.044905

\section{INTRODUCTION}

After successfully verifying the existence of a new state of matter, the quark-gluon plasma (QGP), experiments at the Relativistic Heavy Ion Collider (RHIC) and the Large Hadron Collider (LHC) are now focused on quantifying the transport properties of the QGP and mapping out the QCD phase diagram. The beam energy scan (BES) program at RHIC in particular is aimed at probing the phase diagram of QCD matter at varying values of the baryon chemical potential. It is known that at LHC and top RHIC collision energies, the phase transition between QGP and hadronic matter is a crossover $[1,2]$. However, it is not yet known if there exists a critical point marking the change to first-order phase transition at lower collision energies, where the QCD matter is exposed to typically lower temperatures but higher net-baryon densities.

The beam energy scan has introduced new challenges to the theoretical modeling of relativistic heavy-ion collisions, as the initial nonequilibrium evolution of the system leading up to thermalization gains in importance and has a large effect on the outcome of the calculation. In addition, the assumption of a midrapidity plateau does not hold any longer, requiring a full $(3+1)$-D hydrodynamical model to describe the evolution of the medium. Finally, the equation of state needs to accommodate the nonzero baryon chemical potential $\mu_{B}$. To address these challenges, we utilize a hybrid model, which combines a $(3+1)$-D viscous hydrodynamics model for

*jaa49@phy.duke.edu

Published by the American Physical Society under the terms of the Creative Commons Attribution 4.0 International license. Further distribution of this work must maintain attribution to the author $(s)$ and the published article's title, journal citation, and DOI. Funded by $S C O A P^{3}$. the QGP phase of the reaction with a microscopic hadronic transport model that describes the nonequilibrium evolution of the system before QGP formation and after its subsequent decay into final-state hadrons.

In recent years, a lot of progress has been made in the field regarding the quantification of the shear viscosity over entropy ratio $\eta / s$ in QCD medium. In particular, the temperature dependence of $\eta / s$ has been studied in detail [3-7]. However, it was suggested in a recent study [8] that larger values of the effective shear viscosity are needed to describe the data at lower collision energies, indicating an additional dependence on baryon chemical potential $\mu_{B}$, as earlier calculations had already found to be the case in the hadron resonance gas $[9,10]$.

Note that it was already laid out in Ref. [8] that its scope did not allow for a full uncertainty quantification or study of correlations among the extracted parameters: The parameters were determined by a simple visual fit to data, and the sensitivity of the results on the change in model parameters was examined by varying only one parameter at a time, while others were kept on their default values. While these issues are certainly not unique to that study, they imply that the observed values of $\eta / s$ and their collision energy dependence in general require verification through a more rigorous statistical analysis.

Inspired by the success of Bayesian analysis at LHC energies [6], we re-examine in this article the possible collision energy dependence of $\eta / s$, improving on the analysis presented in Ref. [8] by introducing robust, state-of-the-art statistical analytical methods which allow us to perform the model-todata calibration for all model parameters simultaneously. In addition to ensuring that we find the most likely combination of input parameters, the Bayesian statistical method also provides us the full uncertainty quantification in the form of posterior probability distributions for all the parameters.

In addition to the more sophisticated model-to-data comparison, our current study also benefits from the inclusion of 
the new experimental results on Hanbury-Brown-Twiss (HBT) radii [11] and identified particle multiplicities [12].

We describe the main features and the input parameters of the hybrid model in Sec. II. A summary of the statistical analysis procedure is provided in Sec. III and the details of the simulation setup are found in Sec. IV. Results of the analysis are presented in Sec. V. We summarize our findings in Sec. VI.

\section{HYBRID MODEL}

The hybrid model utilized in our study is the same as in Ref. [8]. In this model, the initial nonequilibrium evolution of the system is modeled with the ultrarelativistic quantum molecular dynamics (UrQMD) hadron-string cascade [13,14], which is known to produce a good description of particle rapidity distributions in heavy-ion collisions at the Super Proton Synchrotron (SPS) and Alternating Gradient Synchrotron (AGS) energies $[15,16]$. As mentioned in the previous section, the longitudinal structure becomes important at the lower end of the beam energy scan range, making ultrarelativistic quantum molecular dynamics (UrQMD) a natural choice for the description of the initial pre-equilibrium dynamics for BES studies.

The earliest possible starting time for a transport-to-hydro transition ("fluidization") is when the two colliding nuclei have passed through each other: $\tau_{0} \geqslant \frac{2 R_{\text {nucleus }}}{\sqrt{\gamma_{C M}^{2}-1}}$. At the transportto-hydro transition, the microscopic particle properties (energy, baryon number) are mapped to hydrodynamic energy, momentum, and charge densities using 3-D Gaussians with "smearing" parameters $W_{\text {trans }}, W_{\text {long }}(\equiv \sqrt{2} \sigma)$. From the hydrodynamic side, cells with sufficiently high local energy density correspond to regions of matter in the QGP phase.

Energy and momentum are conserved in the fluidization procedure, while the smearing parameters control the geometrical volume over which the energy and momentum deposition from each particle is distributed. As the volume increases, the total entropy deposition from each particle to the fluid also increase. Thus, $W_{\text {trans }}$ and $W_{\text {long }}$ regulate the initial entropy in the hydrodynamic stage and will consequently affect the final particle multiplicity.

The hydrodynamic evolution is modeled with $(3+1)-\mathrm{D}$ relativistic viscous hydrodynamics [17]. We utilize a constant value of $\eta / s$ (an input parameter of the model) throughout this stage, which covers the QGP phase of the evolution. Thus the parameter $\eta / s$ in this study represents mainly the effective shear viscosity in QGP, while the total shear viscosity of the system receives additional contribution from the hadron transport. The use of constant $\eta / s$ allows for direct verification of the results presented in Ref. [8]. Furthermore, using a more generic functional form $\eta / s\left(T, \mu_{B}\right)$ would introduce multiple additional parameters, increasing the uncertainties for all parameters in the model-to-data calibration. We believe it is better to first verify if a collision energy dependence on the effective value of $\eta / s$ is observed at all, before attempting to implement a more complex description of the shear viscosity.

In an event-by-event hydrodynamic calculation at the given energy range, temperature as well as baryon chemical potential vary significantly in spacetime. Therefore, an equation of state which covers the large- $\mu_{B}$ sector is a necessary ingredient for the hydrodynamic stage. For the present study, a chiral model equation of state [18] is used. In this equation of state, the transition between hadron and quark-gluon phases is a crossover for all $\mu_{B}$.

The transition from hydrodynamics back to microscopic nonequilibrium transport ("particlization") is performed when the local rest-frame energy density $\epsilon$ in the hydro cells falls below a specified switching value $\epsilon_{\mathrm{SW}}$. The isoenergy density hypersurface is constructed using the Cornelius routine [19]. At this hypersurface, hadrons are sampled via Monte Carlo procedure using Cooper-Frye prescription and including viscous corrections to their distribution functions. The generated hadrons are then fed into the UrQMD cascade to simulate final-state hadronic rescatterings and resonance decays.

It should be noted that before and at the moment of particlization a perfect chemical equilibrium is assumed and thus the transition from hydrodynamics to hadron transport should be performed in sufficiently high energy densities where such assumption is valid. Naturally, the requirement of the chemical equilibrium is relaxed in the hadron transport following the particlization.

\section{STATISTICAL ANALYSIS}

The basics of Bayesian analysis procedure and model emulation have been described in detail in Refs. [20,21]. In this section, we summarize the main points and describe the details specific to the current implementation.

\section{A. Bayes's theorem}

Given a set $X=\left\{\vec{x}_{k}\right\}_{k=1}^{N}$ of points in parameter space (design points), and a corresponding set $Y=\left\{\vec{y}_{k}\right\}_{k=1}^{N}$ of points in observable space (output points), Bayes's theorem states that the conditional probability distribution (posterior) of an input parameter combination $\vec{x}^{*}$, given the observation $\left(X, Y, \vec{y}^{\exp }\right)$, is

$$
P\left(\vec{x}^{*} \mid X, Y, \vec{y}^{\exp }\right) \propto P\left(X, Y, \vec{y}^{\exp } \mid \vec{x}^{*}\right) P\left(\vec{x}^{*}\right),
$$

where $P\left(\vec{x}^{*}\right)$ is the prior probability distribution of $\vec{x}^{*}$ and $P\left(X, Y, \vec{y}^{\exp } \mid \vec{x}^{*}\right)$ is the likelihood of the observation $\left(X, Y, \vec{y}^{\exp }\right)$ for a given $\vec{x}^{*}$.

As in Ref. [21], the likelihood in our analysis will be of the form

$$
P\left(X, Y, \vec{y}^{\exp } \mid \vec{x}^{*}\right) \propto e^{-\frac{1}{2}\left(\vec{y}^{*}-\vec{y}^{\exp }\right)^{T} \Sigma^{-1}\left(\vec{y}^{*}-\vec{y}^{\exp }\right)},
$$

where $\vec{y}^{*}$ is model output for the input parameter point $\vec{x}^{*}$, $\vec{y}^{\exp }$ is the target value, and $\Sigma$ is the covariance matrix.

\section{B. Gaussian emulator}

To calculate the likelihood, one must be able to determine the model output $\vec{y}^{*}$ for an arbitrary $\vec{x}^{*}$. As the simulations typically take several hours to run, it is highly impractical to run the full model during statistical analysis. Instead, the model is emulated with a Gaussian process. That is, the model is taken as a mapping of a set $X$ of points in the parameter space to a set $Y_{a}$ of values of the observable $y_{a}$,

$$
F: X \rightarrow Y_{a},
$$

where $Y_{a}$ is assumed to have a multivariate normal distribution

$$
Y_{a} \sim \mathcal{N}\left[\mu(X), \Sigma_{X, X}\right]
$$


with $\mu(X)=\left\{\mu\left(\vec{x}_{1}\right), \ldots, \mu\left(\vec{x}_{N}\right)\right\}$ as the mean and

$$
\Sigma_{X, X}=\left(\begin{array}{ccc}
\sigma\left(\vec{x}_{1}, \vec{x}_{1}\right) & \cdots & \sigma\left(\vec{x}_{1}, \vec{x}_{N}\right) \\
\vdots & \ddots & \vdots \\
\sigma\left(\vec{x}_{N}, \vec{x}_{1}\right) & \cdots & \sigma\left(\vec{x}_{N}, \vec{x}_{N}\right)
\end{array}\right)
$$

as the covariance matrix with covariance function $\sigma\left(\vec{x}, \vec{x}^{\prime}\right)$. The covariance matrix is further reformulated in terms of a triangular matrix $S$ using the Cholesky decomposition $\Sigma=S S^{T}$. A sample from a Gaussian process is then of the form

$$
\vec{y}_{G P}(\vec{x})=\mu(\vec{x})+S \vec{u},
$$

where the components of $\vec{u}$ are normally distributed random variables, $u_{i} \sim N(0,1)$.

To predict the model output $y_{0}$ in an arbitrary point $\vec{x}_{0}$, one writes the joint distribution

$$
\left(\begin{array}{c}
y_{0} \\
Y
\end{array}\right)=\mathcal{N}\left[\left(\begin{array}{c}
\mu\left(\vec{x}_{0}\right) \\
\mu(X)
\end{array}\right),\left(\begin{array}{cc}
\Sigma_{0,0} & \Sigma_{0, X} \\
\Sigma_{X, 0} & \Sigma_{X, X}
\end{array}\right)\right]
$$

and calculates the conditional predictive mean

$$
\bar{\mu}\left(\vec{x}_{0}\right)=\mu\left(\vec{x}_{0}\right)+\Sigma_{0, X} \Sigma_{X, X}^{-1}[Y-\mu(X)] .
$$

Typically, we define the mean function $\mu(\vec{x}) \equiv 0$ and the prediction is simply

$$
\mu\left(\vec{x}_{0}\right)=\Sigma_{0, X} \Sigma_{X, X}^{-1} Y
$$

with the associated predictive (co)variance

$$
\bar{\Sigma}=\Sigma_{0,0}-\Sigma_{0, X} \Sigma_{X, X}^{-1} \Sigma_{X, 0} .
$$

Clearly the crucial component of the Gaussian process is the covariance matrix $\Sigma$. The covariance function $\sigma\left(\vec{x}, \vec{x}^{\prime}\right)$ can have any form from a simple constant to an exponential or a periodic function, and the right choice depends on the features of the emulated model. For this study, we have chosen the squared-exponential covariance function with a noise term

$$
\sigma\left(\vec{x}, \vec{x}^{\prime}\right)=\theta_{0} \exp \left[-\sum_{i=1}^{n} \frac{\left(x_{i}-x_{i}^{\prime}\right)^{2}}{2 \theta_{i}^{2}}\right]+\theta_{\text {noise }} \delta_{\vec{x} \vec{x}^{\prime}} .
$$

The hyperparameters $\vec{\theta}=\left(\theta_{0}, \theta_{1}, \ldots, \theta_{n}, \theta_{\text {noise }}\right)$ are estimated from the provided data $(X, Y)$. This "training" of the emulator is done by maximizing the multivariate Gaussian log-likelihood function

$$
\begin{aligned}
\log P(Y \mid X, \vec{\theta})= & -\frac{1}{2} Y^{T} \Sigma_{X, X}^{-1}(\vec{\theta}) Y \\
& -\frac{1}{2} \log \left|\Sigma_{X, X}(\vec{\theta})\right|-\frac{N}{2} \log (2 \pi) .
\end{aligned}
$$

In principle, using maximum likelihood values for hyperparameters is only an approximative method, as in the rigorous Bayesian approach the hyperparameters are allowed to change during the calibration procedure, resulting to posterior distributions also for the hyperparameters. However, in practice 100 training points create strong enough constraints on the Gaussian processes that the precise value of the hyperparameters has little effect on the final results.

\section{Principal component analysis}

Generally, the analysis requires one emulator per data point. However, the number of emulators can be reduced with a principal component analysis, which is an orthogonal linear transformation of the data onto the directions of maximal variance.

We use our $N$ simulation points and $m$ observables to form a $N \mathrm{x} m$ data matrix $Y$. Data are normalized with the corresponding experimental values to obtain dimensionless quantities and centered by subtracting the mean of each observable from all points. The singular value decomposition of $Y$ is

$$
Y=U S V^{T}
$$

where $S$ is a diagonal matrix containing the singular values, and $U$ and $V^{T}$ are orthogonal matrices containing the left- and right-singular vectors, respectively. Eigenvalue decomposition of the covariance matrix $Y^{T} Y$ becomes

$$
Y^{T} Y=V S^{2} V^{T} .
$$

Singular values in $S$ are square roots of eigenvalues $\lambda_{i}$, and right singular vectors $\vec{v}_{i}$ represented by columns in $V^{T}$ are eigenvectors of $Y^{T} Y$; these are the principal components (PCs). The eigenvalues are proportional to the total variance of the data, such that the eigenvalue of the first principal component explains the largest fraction of the variance.

We can now write our data matrix in principal component space:

$$
Z=\sqrt{N} Y V .
$$

Since $\lambda_{1} \geqslant \lambda_{2} \geqslant \cdots \geqslant \lambda_{m}$, the fraction of the total variance explained by the $q$ th principal component, $\lambda_{q} /\left(\sum_{j=1}^{m} \lambda_{j}\right)$, becomes negligible starting from some index $q<m$. This allows us to define a lower-rank approximation of the original $Z$ as $Z_{q}=\sqrt{N} Y V_{q}$.

In addition to dimensional reduction, we perform data whitening, i.e., divide each principal component with their singular value such that they all have unit variance. The full prescription for transforming a vector $\vec{y}$ from observable space to vector $z$ in whitened principal component space is thus defined as

$$
\vec{z}=\vec{y} \tilde{V}_{q} \equiv \vec{y} \sqrt{N} V_{q} S_{q}^{-1} .
$$

To compare an emulator prediction $\vec{z}^{*}$ against physical observables, we use inverse transformation

$$
\vec{y}^{*}=\vec{z}^{*} \frac{1}{\sqrt{N}} S_{q} V_{q}^{T} .
$$

\section{Likelihood function}

The log-likelihood function used for determining the posterior probability distribution in this analysis has the following form:

$$
\begin{aligned}
\log P\left(\vec{z}^{\exp } \mid \vec{x}^{*}\right)= & -\frac{1}{2}\left(\vec{z}^{*}-\vec{z}^{\exp }\right) \Sigma_{z}^{-1}\left(\vec{z}^{*}-\vec{z}^{\exp }\right)^{T} \\
& -\frac{1}{2} \log \left|2 \pi \Sigma_{z}\right|,
\end{aligned}
$$

where $\vec{z}^{*}$ is the emulator prediction at the input parameter point $\vec{x}^{*}$ and $\vec{z}^{\exp }$ represents the experimental data transformed to principal component space. 
The covariance matrix includes both experimental and emulator uncertainties: $\Sigma_{z}=\Sigma_{z}^{\exp }+\Sigma_{z}^{\mathrm{GP}}$. The uncertainty of experimental data $\Sigma_{z}^{\exp }$ is obtained by transforming the observable space covariance into principal component space: ${ }^{1}$

$$
\Sigma_{z}^{\exp }=\tilde{V}_{q}^{T} \Sigma_{y}^{\exp } \tilde{V}_{q}=\tilde{V}_{q}^{T} \operatorname{diag}\left(\sigma_{0}^{2}, \ldots, \sigma_{m}^{2}\right) \tilde{V}_{q} .
$$

For many of the observables, the reported values $\sigma_{0}, \ldots, \sigma_{m}$ include both statistical and systematic uncertainties. In cases where the uncertainties are reported separately, they have been added quadratically: $\sigma_{i}^{2}=\sigma_{i \text {,stat }}^{2}+\sigma_{i, \text { sys }}^{2}$. In principle, systematic uncertainties introduce also off-diagonal terms into $\Sigma_{y}^{\exp }$. However, the task of estimating these off-diagonal terms and investigating their effect on the final results requires detailed knowledge of the detector systems in the experiments and is referred to a future study.

The emulator uncertainty is included as

$$
\Sigma_{z}^{\mathrm{GP}}=\operatorname{diag}\left(\sigma_{z 0}^{2}, \ldots, \sigma_{z q}^{2}\right),
$$

where $\sigma_{z i}^{2}$ refers to the GP predictive variance [Eq. (10)] for the $i$ th principal component. Finally, the term containing the determinant of the total covariance matrix $\left|2 \pi \Sigma_{z}\right|$ is included to provide more value for points which both fit the data and have small uncertainties, as opposed to points which fit the data but have large uncertainties.

\section{E. Data calibration using random walkers}

The posterior distribution is sampled using the Markov chain Monte Carlo (MCMC) method, which is a random walk in parameter space, where each step is accepted or rejected based on a relative likelihood. It converges to posterior distribution at the limit $N_{\text {steps }} \rightarrow \infty$.

An important measure for the quality of the resulting random walk is the acceptance fraction, which should be between 0.2 and 0.5 [25]. A very low value of the acceptance fraction indicates that the walkers are stuck, while values close to 1 mean all proposals are accepted and the walk is purely random.

Another measure is the autocorrelation time, which is an estimate of the number of steps required between independent samples. This can be used to evaluate if the random walk contained enough steps to produce a proper sample size.

We initialize $\mathcal{O}(1000)$ random walkers in random positions in the input parameter space. This is followed by the "burnin" phase, which allows the random walkers to converge to the high-likelihood areas. At the end of the burn-in, the samples produced during this phase are removed and the proper sampling phase is initialized.

Once the MCMC is complete, we verify that we had sufficient number of steps in the burn-in phase to cover a couple autocorrelation lengths, and enough steps in the sampling phase to cover $\sim \mathcal{O}(10)$ autocorrelations. Having thousands

${ }^{1}$ In the previous Bayesian beam energy scan analyses [22-24], the experimental uncertainties were treated as simple diagonal contributions in the principal component space: $\Sigma_{z}^{\exp }=\operatorname{diag}\left(\left(\sigma_{0}^{\prime}\right)^{2}, \ldots,\left(\sigma_{q}^{\prime}\right)^{2}\right)$, where $\sigma^{\prime}=\left(\sigma_{0}, \ldots, \sigma_{m}\right) \tilde{V}_{q}$. However, this neglected the covariances between the principal components and exaggerated the variance for the first principal component. The uncertainties were overestimated as a net result. of walkers ensures a sufficiently large sample of independent observations for generating posterior distributions.

\section{SIMULATION SETUP}

\section{A. Input parameters}

The parameters of the computational model encode the physical properties of the system that we wish to extract from our analysis. These are the effective shear viscosity of the QGP $\eta / s$, the thermalization time of the system $\tau_{0}$, the initial condition smearing parameters $W_{\text {trans }}$ and $W_{\text {long }}$, as well as the hydro-to-transport transition energy density that can provide information on the temperature scale at which nonequilibrium effects in the hadronic evolution become important. The following parameter ranges were used for the design points:

(1) Effective shear viscosity over entropy density $\eta / s$ during hydrodynamical evolution: $0.001-0.3$ (extended to 0.4 for $\sqrt{s_{N N}}=19.6 \mathrm{GeV}$.)

(2) Transport-to-hydro transition time $\tau_{0}: 0.4-3.1 \mathrm{fm} / c$.

(3) Transverse Gaussian smearing of particles $W_{\text {trans }}: 0.2$ $2.2 \mathrm{fm}(19.6 \mathrm{GeV}), 0.4-2.2 \mathrm{fm}(39 \mathrm{GeV}), 0.5-2.1 \mathrm{fm}$ $(62.4 \mathrm{GeV})$.

(4) Longitudinal Gaussian smearing of particles $W_{\text {long }}$ : $0.2-2.2 \mathrm{fm}(19.6 \mathrm{GeV}), 0.4-2.2 \mathrm{fm}(39 \mathrm{GeV}), 0.5-2.1$ fm $(62.4 \mathrm{GeV})$.

(5) Hydro-to-transport transition energy density $\epsilon_{\mathrm{SW}}$ : $0.15-0.75 \mathrm{GeV} / \mathrm{fm}^{3}$.

As described in Sec. II, $\tau_{0}$ is constrained by the condition that the two nuclei must have passed each other, and thus the lower limit depends on collision energy:

$$
\tau_{0} \geqslant 2 R / \sqrt{s_{N N} /\left(2 m_{N}\right)^{2}-1} .
$$

The cells in the hydro grid have a transverse length $\Delta x=$ $\Delta y=0.25 \mathrm{fm}$ and a longitudinal length $\Delta \eta=0.15$, which sets a soft lower limit for the smearing parameters, as the limited resolution will lead to the "oversmearing" of the Gaussians much narrower than the cell size.

As in Ref. [21], the input parameter combinations were sampled using the maximin Latin hypercube method, which attempts to optimize the sample by maximizing the minimum distance between the design points.

\section{B. Normality of the data}

Principal component analysis assumes that variance provides a reasonable measure of the spread of the simulation output values; i.e.. the simulation data are approximately normally distributed. However, this is not generally true for the distributions of observable values calculated from the model output. We aim to normalize the distributions by scaling the output with the corresponding experimental data and applying the Box-Cox transformation [26] on these dimensionless values $\tilde{y}=y / y^{\exp }$ :

$$
y^{(\lambda)}=\left\{\begin{array}{l}
\left(\tilde{y}^{\lambda}-1\right) / \lambda: \lambda \neq 0 \\
\log \tilde{y}: \lambda=0
\end{array} .\right.
$$

We check the normality of the resulting distribution with a probability plot, which is a method for comparing the quantiles 
of the sample data against the respective quantiles of the normal distribution. We perform a least-squares fit on the points in probability plot: The goodness of the fit can be estimated by the coefficient of determination $R^{2}$, which should be close to 1. We use $R_{c}^{2}=0.91$ as a (very lenient) critical test value for deviations from normal distribution that are too large. Observables with $R^{2}<R_{c}^{2}$ are excluded from the analysis.

\section{Event selection}

Centrality bins were determined based on the number of participants $N_{\text {part }}$, such that the average number of participants $\left\langle N_{\text {part }}\right\rangle$ in each bin matches the respective experimental estimate [12,27-30].

To save computation time, the centrality selection was done by running the full simulation for the $50 \%$ of the initial UrQMD events with highest number of participants $N_{\text {part }}$, instead of running the full simulation for all initial states and sorting the events by final charged particle multiplicity $N_{\mathrm{ch}}$. In addition, each switching energy density hypersurface has been used 50 times for particle sampling ("oversampling") to increase statistics. As the number of independent hypersurfaces in any centrality bin is at least an order of magnitude greater, this method is unlikely to produce any notable bias in the final results.

\section{RESULTS}

\section{A. Emulator verification}

The quality of the GP emulator was verified against six randomly selected test points from the simulation output data, which were not included in the analysis. As an example, Fig. 1 shows the verification results on $\pi^{+}$yields at $\sqrt{s_{N N}}=$ $19.6 \mathrm{GeV}$. The untrained emulator is typically able to make reasonably accurate predictions already, which indicates that the GPs are already considerably constrained by the density of design points in the input parameter space. After training, the agreement with the test point values is very good. This assures us that the principal components of the model variance have been correctly identified and the chosen kernel for Gaussian processes provides a good description of the model behavior with respect to the principal components.

\section{B. Presentation of analytical results}

The Bayesian posterior probability distributions for $\sqrt{s_{N N}}=19.6,39$, and $62.4 \mathrm{GeV}$ are illustrated in Figs. 2, 12 , and 20, respectively, and will be discussed in detail in the following sections. We visualize these five-dimensional probability distributions using corner plots. The histograms on the diagonal panels represent the probability distributions of each input parameter integrated over the possible values of the other four parameters (also known as marginal distributions), while the off-diagonal panels show pairwise correlations between the parameters.

Model results after the calibration are compared to experimental data in the following way: To illustrate how the uncertainty in parameter values reflects to the observables, 100 random parameter combinations were drawn from the posterior and the model outputs for these combinations were predicted by the Gaussian process emulator. These emulator predictions are drawn as box-and-whisker plots. The single red horizontal
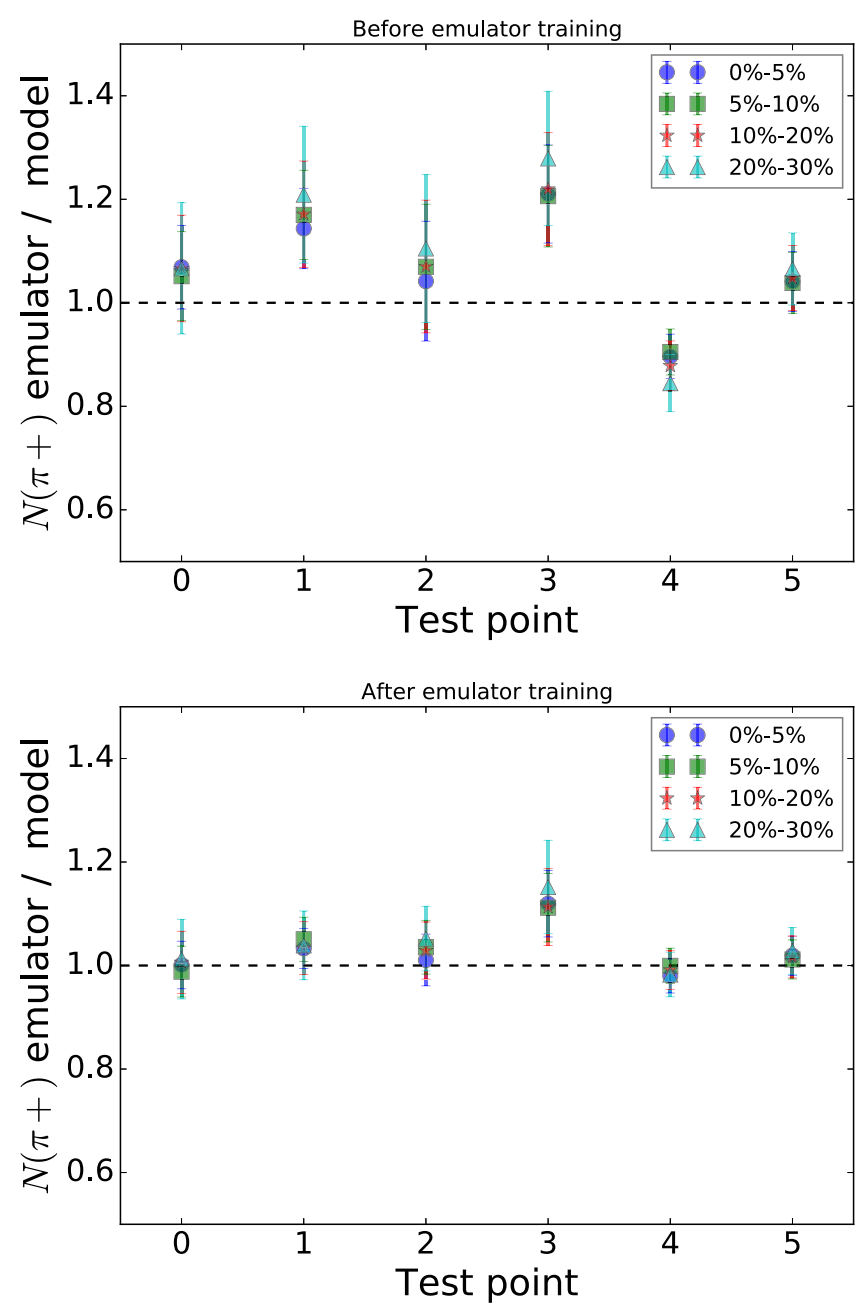

FIG. 1. The ratio of the Gaussian process prediction over the model result for positively charged pion yields, for the six test points at $\sqrt{s_{N N}}=19.6 \mathrm{GeV}$. Top panel: Untrained emulator. Bottom panel: After emulator training.

line within each box represents the median prediction; that is, half of the predictions are smaller and half are larger than this value. Boxes represent the values between first and third quartiles $Q_{1}$ and $Q_{3}$; this means $25 \%$ of the predictions were smaller than the values in the box and $25 \%$ were larger. Finally, the whiskers represent either the complete range of predictions, or 1.5 times the interquartile range $Q_{3}-Q_{1}$, whichever is smaller. In the latter case, the predictions outside the whisker range ("outliers") are represented by individual points. Thus, the box-and-whisker representation illustrates the shape of the distribution of emulator predictions.

To verify the quality of the calibration, full model simulations were run with the median values of the one-dimensional projections of each posterior probability distribution, represented as dashed red lines and red triangles in the aforementioned corner plots.

\section{C. $\sqrt{s_{N N}}=19.6 \mathrm{GeV}$}

The posterior distribution for $\sqrt{s_{N N}}=19.6 \mathrm{GeV}$ is presented in Fig. 2. While the data support the earliest possible 


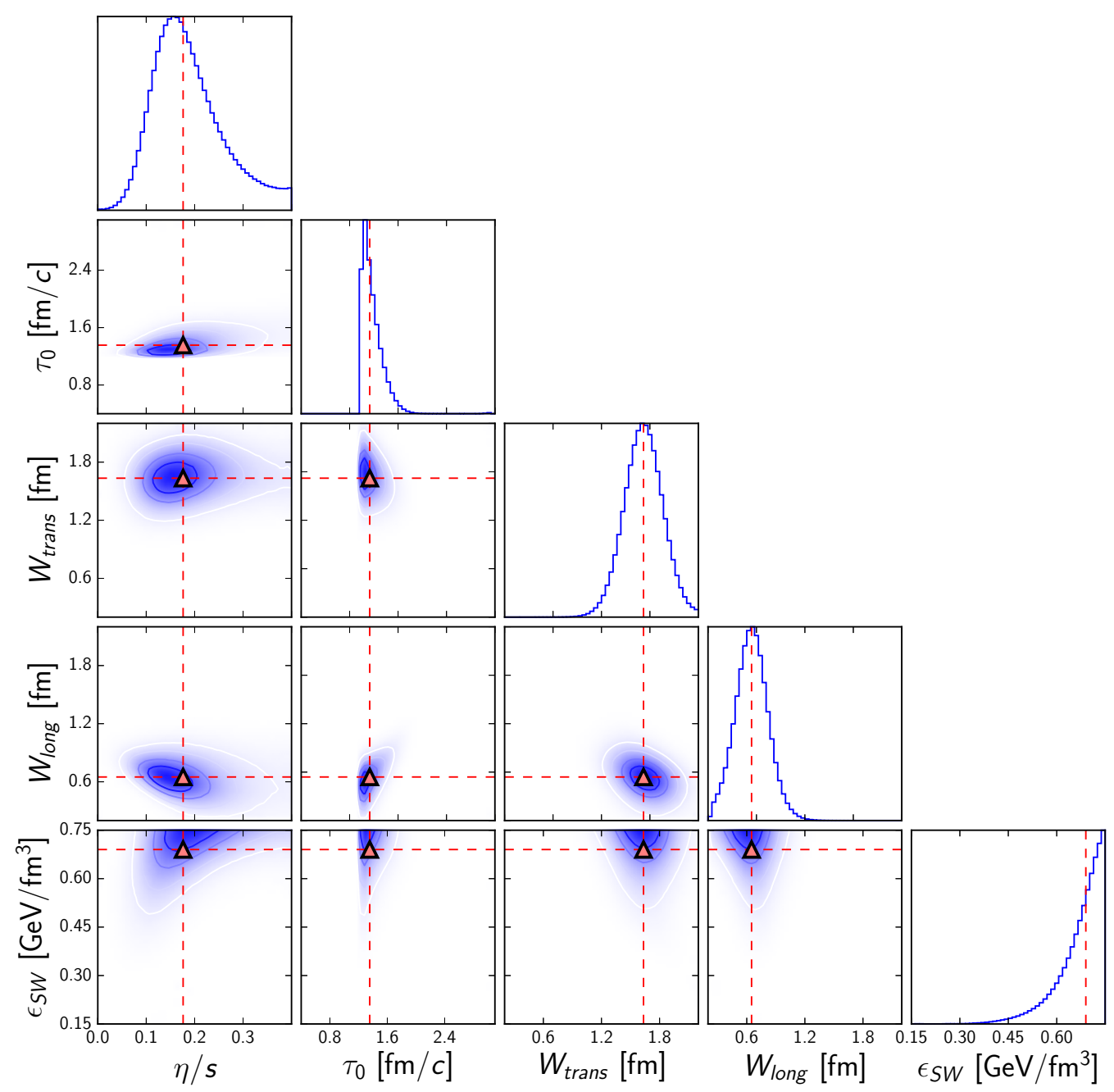

FIG. 2. Representation of the posterior probability distribution of the input parameters at $\sqrt{s_{N N}}=19.6 \mathrm{GeV}$. Panels on a diagonal show one-dimensional projections of the posterior for each parameter axis, while the off-diagonal panels display projections on the different 2-D planes of the input parameter space. Dashed red lines indicate median values of the one-dimensional distributions.

starting time and longitudinally narrow Gaussians as density representations of the initial particles, the transverse plane of the initial density profile is strongly smeared. An early transition from hydro to hadron transport is preferred, which is in contrast to the earlier studies where $\Omega$ baryon was given more weight in the analysis [22-24].

Examination of the pairwise correlations of $\eta / s$ with the four "nuisance parameters" $\tau_{0}, W_{\text {trans }}, W_{\text {long }}$, and $\epsilon_{\mathrm{SW}}$ suggests that to a small extent around the most probable value, a change in the effective shear viscosity (increase or decrease) can be compensated by an opposite change in $W_{\text {long }}$. Increasing $W_{\text {long }}$ weakens the longitudinal expansion and increases the initial entropy. Hence, the observed anticorrelation between longitudinal smearing and shear viscosity is most likely driven by the pseudorapidity distribution and the overall charged particle multiplicity.

Based on their effects on flow observables, one would expect to see a negative correlation between shear viscosity and transverse smearing (increase in viscosity could at least partially be compensated by a more granular initial state, or vice versa). This relation between shear viscosity and initial- state fluctuations appears to be diminished by the constraints from other observables at this collision energy, however.

Correlations between $\eta / s$ and $\tau_{0}$ are suppressed by the strong overall constraint on the most likely transport-to-hydro switching time. The situation is similar for the switching energy density $\epsilon_{\mathrm{SW}}$, which has its probability density strongly concentrated at larger values of the prior.

We can investigate the quality of statistical analysis in more detail by looking the results for each observable in turn. In Figs. 3 and 4, results from simulations using the posterior median values are shown for the charged particle pseudorapidity distributions $d N_{\mathrm{ch}} / d \eta$ for (0-6)\% and (15$25) \%$ centrality at $\sqrt{s_{N N}}=19.6 \mathrm{GeV}$. For the purposes of the statistical analysis, the pseudorapidity distribution was reduced to two descriptive numbers: the value of $d N / d \eta$ at $\eta=0.1$ bin and the average $\langle\eta\rangle$ over the positive part of the distribution.

We can observe from Fig. 3 that the midrapidity modelto-data agreement is very good when using the posterior median. Emulator predictions (black open boxes) appear to underestimate the real simulation result (red triangles). As will become clear, the pseudorapidity distribution is one of the rare 

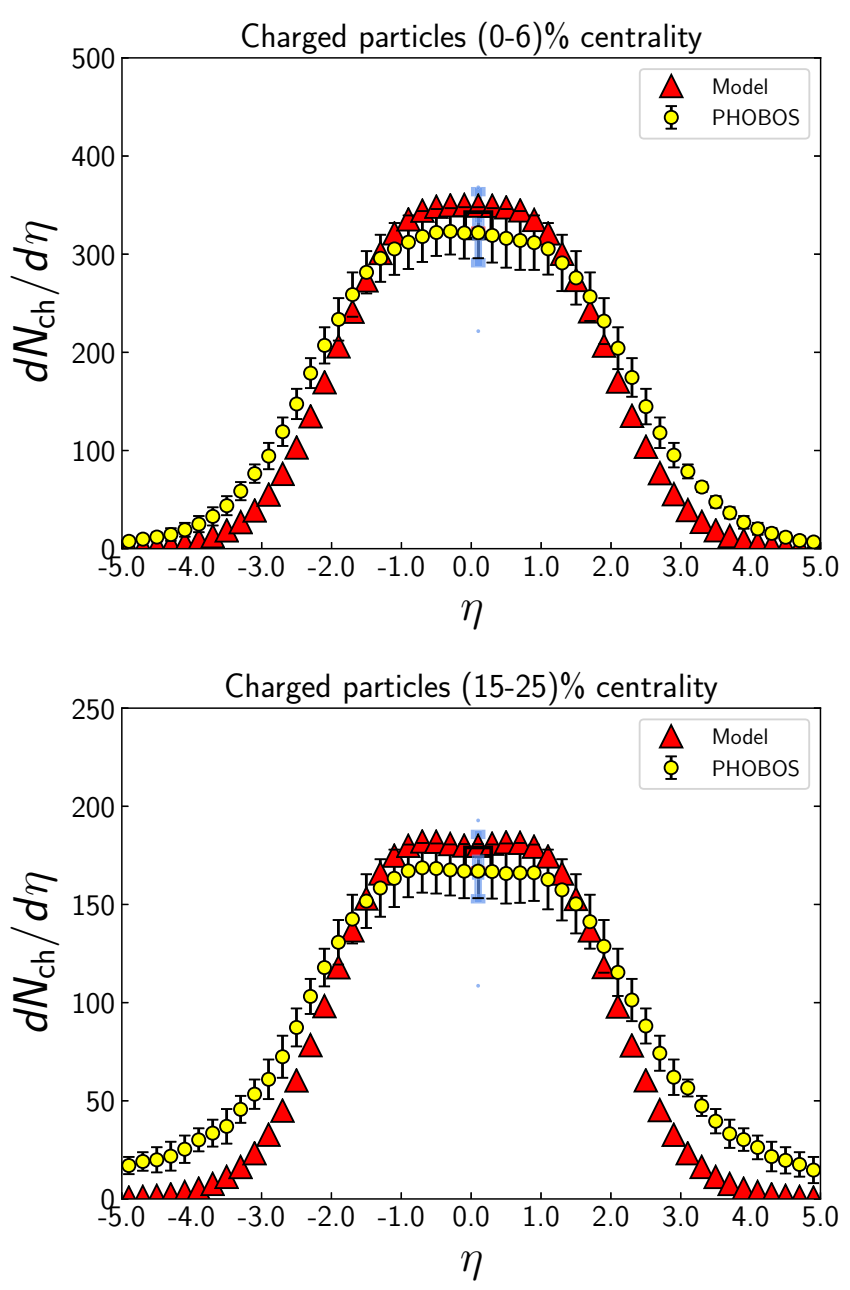

FIG. 3. Charged particle pseudorapidity distribution at $\sqrt{s_{N N}}=$ $19.6 \mathrm{GeV}$ for (0-6)\% centrality (top) and (15-25)\% centrality (bottom) from the simulations using the posterior peak values for model parameters. Blue boxes with whiskers represent the range of Gaussian process emulator predictions for 100 samples from the posterior. Black open squares are emulator predictions for the model output at distribution median values (red triangles). PHOBOS data from Ref. [28].

observables where the emulator prediction is systematically off, although still reasonably close to the true value.

At larger pseudorapidities, the longitudinal expansion is systematically underestimated, as Fig. 4 clearly illustrates. The range of values over all training points are represented as dashed boxes in the figure, which lies below the $\langle\eta\rangle$ value calculated from experimental data; thus, it would not be possible to fully match the PHOBOS data with the currently used input parameter priors.

The recently published STAR data on identified particle yields and mean transverse momenta [12] finally makes it possible to rigorously test the bulk production capabilities of relativistic heavy-ion collision models over the whole beam energy scan range. However, as the proton and antiproton data suffer from additional uncertainties related to feed-down corrections, we limit ourselves to pion and kaon data in this analysis.

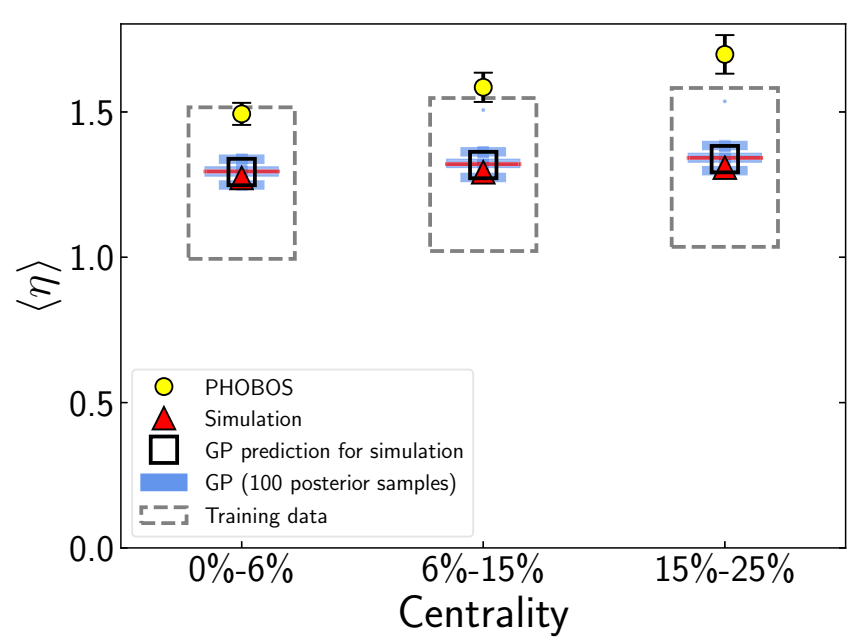

FIG. 4. Centrality dependence of average charged-particle pseudorapidity $\langle\eta\rangle$ for $\eta>0.0$ at $\sqrt{s_{N N}}=19.6 \mathrm{GeV}$. Blue box-whisker plots are GP estimates for samples from the posterior distribution. Black open squares are emulator predictions for the model output at distribution median values (red triangles). Dashed gray boxes represent the range of values from the training data. Experimental values are estimated from PHOBOS data [28].
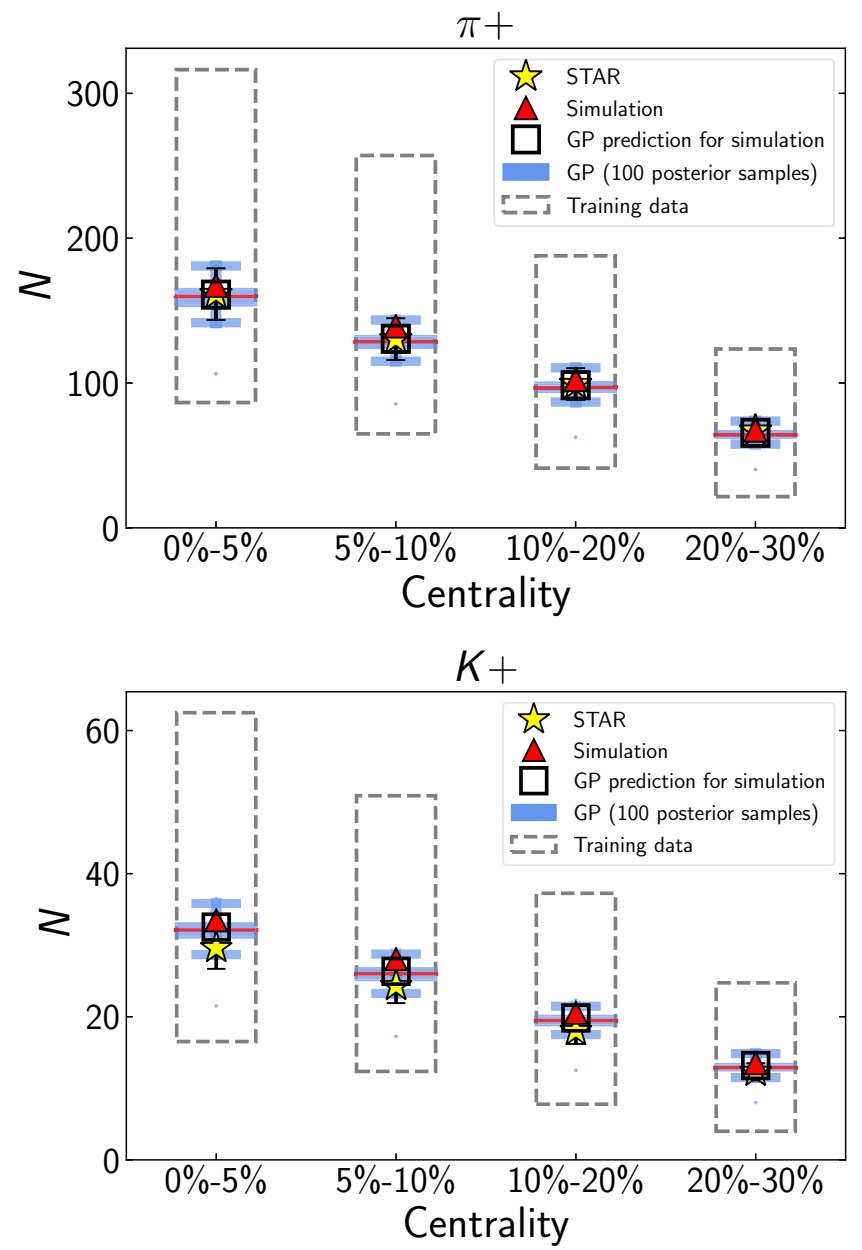

FIG. 5. Multiplicities of $\pi^{+}$and $K^{+}$at midrapidity as a function of centrality at $\sqrt{s_{N N}}=19.6 \mathrm{GeV}$. Experimental data from Ref. [12]. 


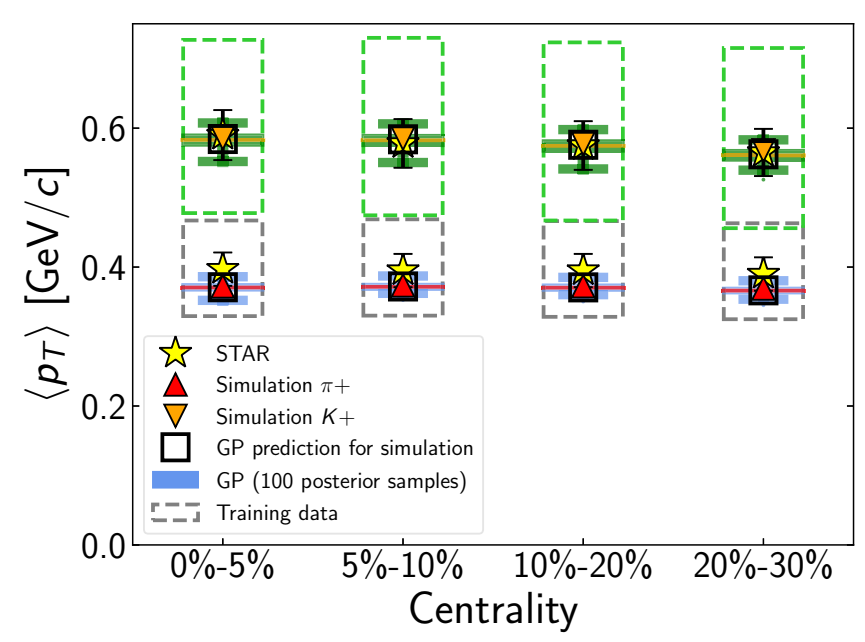

FIG. 6. Mean transverse momentum of $\pi^{+}$(red up triangles) and $K^{+}$(orange down triangles) as a function of centrality at $\sqrt{s_{N N}}=$ 19.6 GeV. Experimental data from Ref. [12].

The results for positive-charge pion and kaon multiplicities are shown in Fig. 5. Pion yields are well reproduced for the investigated centrality range. There is a tendency to overestimate kaon yields, especially at the more central collisions. The median value result is still within statistical uncertainties, however.

To convince us that the model has captured the correct dynamical evolution of the system, it is important to check not only particle yields but also the transverse momentum spectra $d N / d p_{T}$. It was postulated in Ref. [20] that the combination of integrated yield and mean $p_{T}$ is sufficient to describe the $p_{T}$ spectrum. Results for mean transverse momenta of $\pi^{+}$ and $K^{+}$are shown in Fig. 6. The pion transverse momenta is slightly below reported value for all centralities, but still within error bars, whereas the agreement with kaon data is very good. Overall pion and kaon spectra are well reproduced, as shown in

$(0-5) \%$ centrality

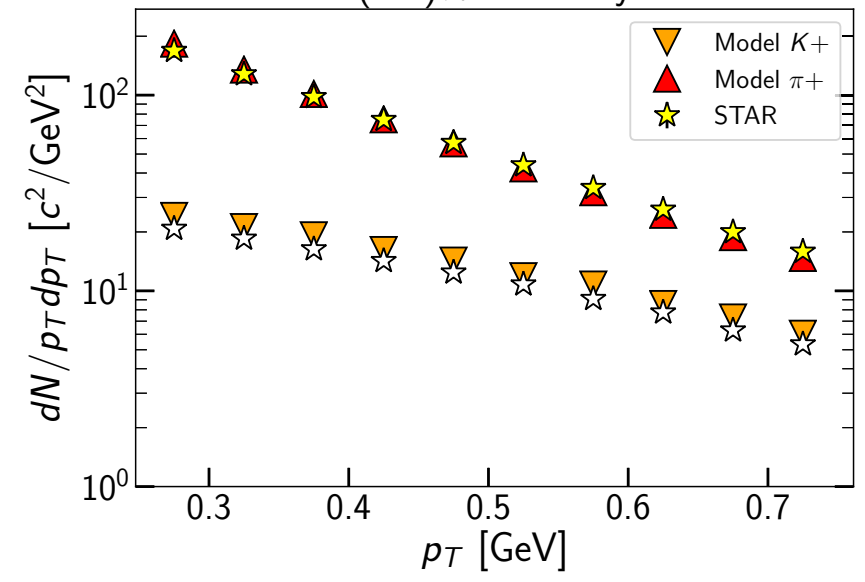

FIG. 7. Transverse momentum spectra of $\pi^{+}$(red up triangles) and $K^{+}$(orange down triangles) for $(0-5) \%$ centrality at $\sqrt{s_{N N}}=$ 19.6 GeV. Experimental data from Ref. [12].

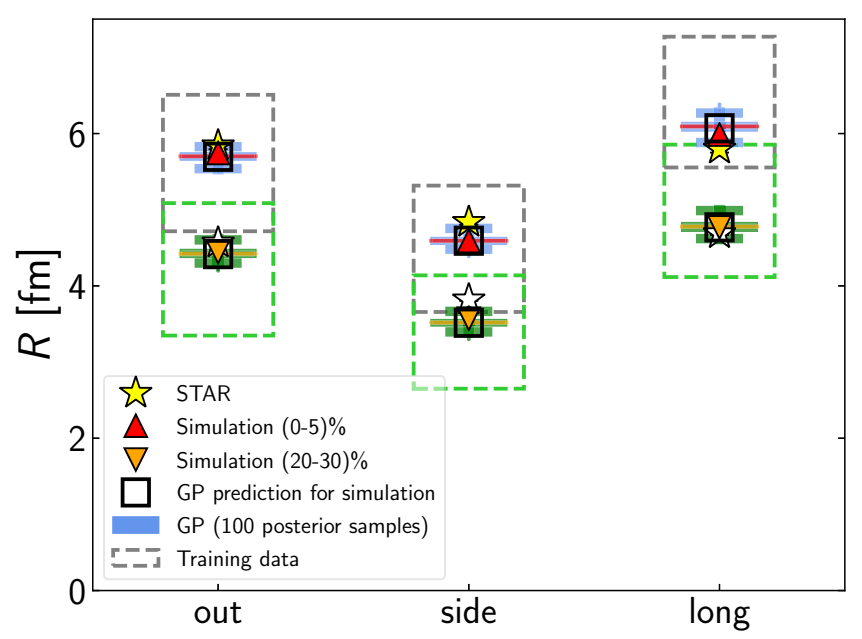

FIG. 8. $R_{\text {out }}, R_{\text {side }}$, and $R_{\text {long }}$ of charged $\pi$ at $\left\langle k_{T}\right\rangle \approx 0.22 \mathrm{GeV} / c$ at $(0-5) \%$ and (20-30)\% centralities (red up triangles and orange down triangles, respectively) at $\sqrt{s_{N N}}=19.6 \mathrm{GeV}$. Experimental data from Ref. [11].

Fig. 7, although the kaon excess in the most central collisions is evident also on this figure.

Two-pion interferometry, commonly referred as "HanburyBrown-Twiss (HBT) analysis," is considered as a probe of the space-time geometry of the kinetic freeze-out region [31]. Results for the HBT radii are summarized in Fig. 8 for the lowest $\left\langle k_{T}\right\rangle$ bin, which corresponds to the observable used for the comparison over several different experiments in Ref. [11]. Again following the example of Ref. [20], we also investigate mean radii averaged over several $k_{T}$ bins in Fig. 9.

Using the median values for input parameters, the model is able to match $R_{\text {out }}$ and $R_{\text {long }}$ quite well, but $R_{\text {side }}$ is systematically underestimated. The same is true for the averaged radii. Emphasizing the importance of HBT radii has thus potential to impose strong constraints on the input parameters.

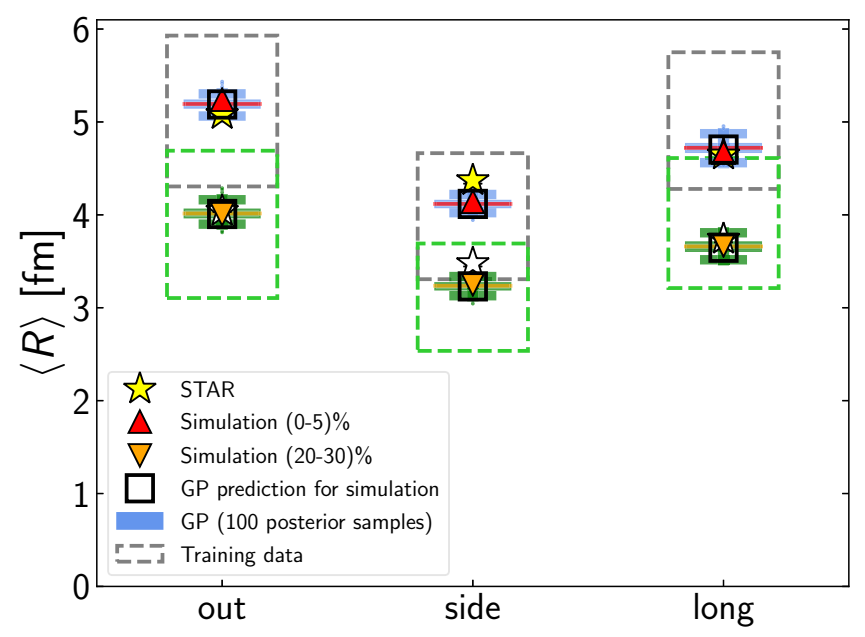

FIG. 9. $R_{\text {out }}, R_{\text {side }}$, and $R_{\text {long }}$ of charged $\pi$ averaged over 0.15 $\mathrm{GeV} / c<k_{T}<0.6 \mathrm{GeV} / c$ at $(0-5) \%$ and $(20-30) \%$ centralities at $\sqrt{s_{N N}}=19.6 \mathrm{GeV}$. Experimental data from Ref. [11]. 


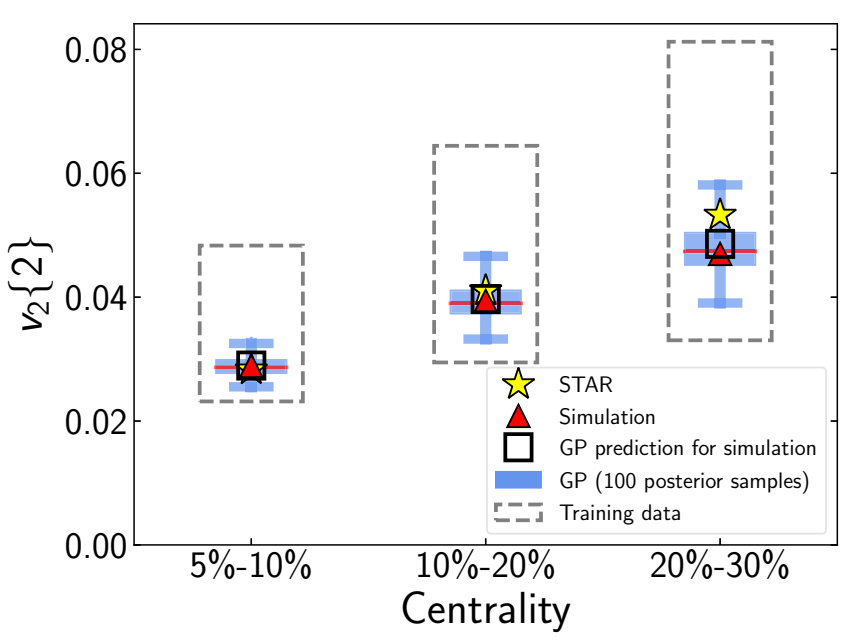

FIG. 10. Centrality dependence of charged particle $v_{2}\{2\}$ at $\sqrt{s_{N N}}=19.6 \mathrm{GeV}$. Experimental data from Ref. [32].

The centrality dependence of $v_{2}\{2\}$ for charged particles is presented in Fig. 10. Agreement with the STAR data is very good for the $(5-10) \%$ and $(10-20) \%$ centralities; in the

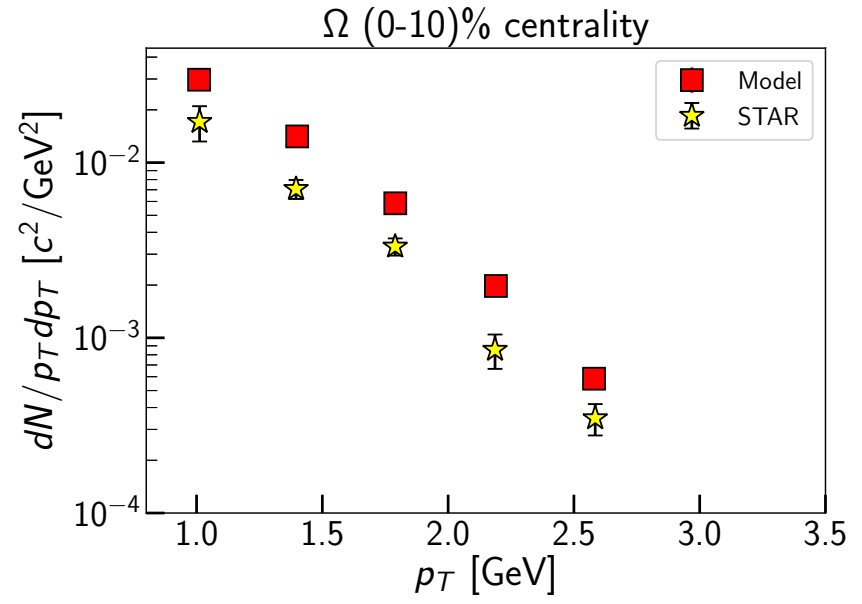

FIG. 11. Transverse momentum spectrum of $\Omega$ at $\sqrt{s_{N N}}=$ 19.6 GeV. Experimental data from Ref. [33].

(20-30)\% centrality bin, the median input parameters produce slightly less elliptic flow compared to observations, which may indicate an issue using the same value of $\tau_{0}$ for all centralities.

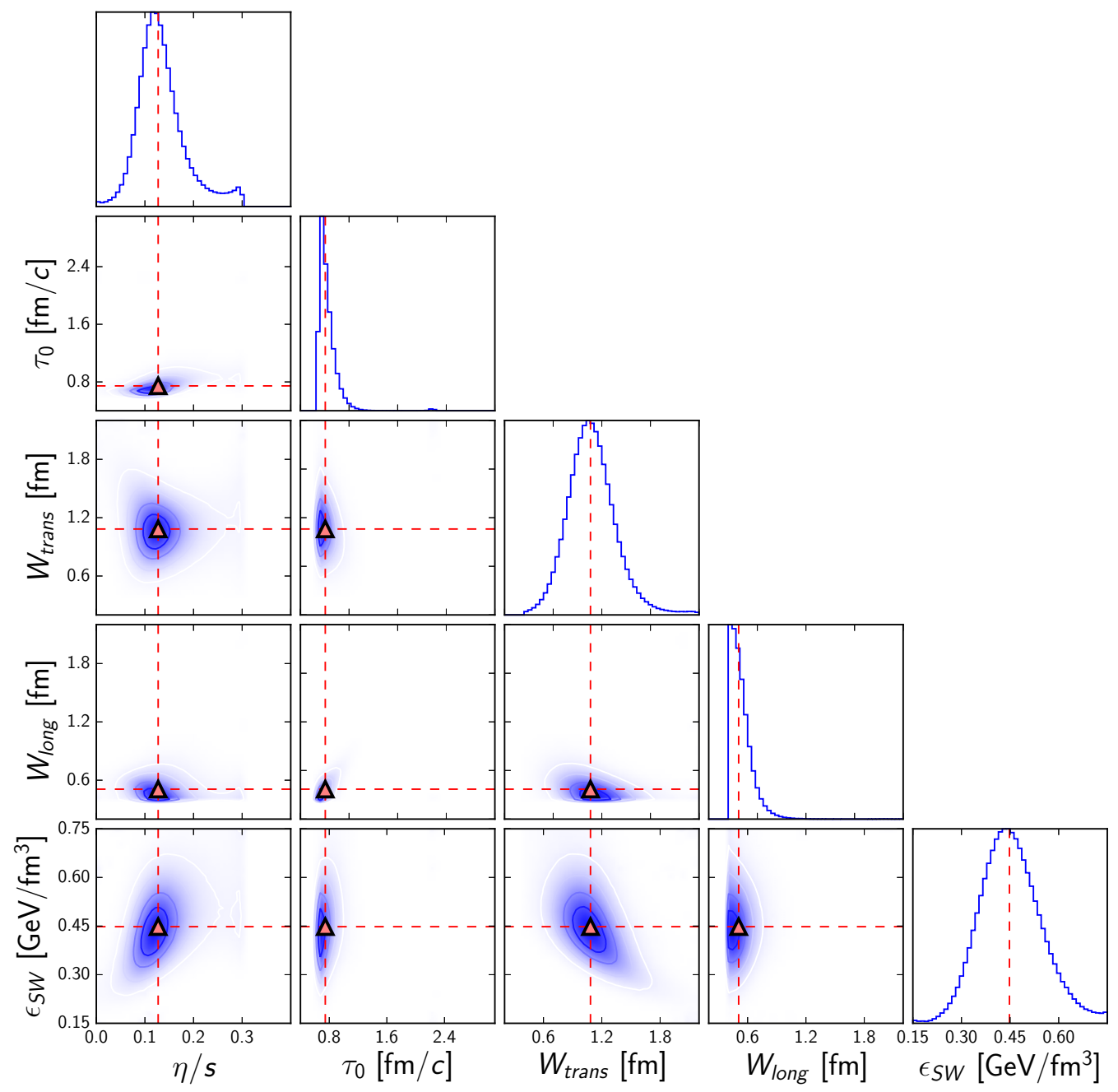

FIG. 12. Representation of the posterior probability distribution of the input parameters at $\sqrt{s_{N N}}=39 \mathrm{GeV}$. See caption of Fig. 2 for details. 
The lower limit of $\tau_{0}$, the time for the two nuclei to pass each other, becomes smaller at higher centralities, in principle enabling earlier hydro starting times. This might also effect longitudinal smearing factor $W_{\text {long }}$ which tends to have strong correlation with $\tau_{0}$. We leave the investigation of the effect of centrality-dependent $\tau_{0}$ for future work.

Finally, we compare the transverse momentum spectrum of $\Omega$ against the STAR data in Fig. 11. It is evident from the figure that $\Omega$ yield is overestimated when using the median values of the posterior distribution. However, the fit here and at $39 \mathrm{GeV}$ was done using the $p_{T}=1.01 \mathrm{GeV} / c$ data point and the estimated mean transverse momentum for the $p_{T}$ range $1-5 \mathrm{GeV} / c$, which was calculated from a Levy fit on the $p_{T}$ spectra [22]. Based on results at $62.4 \mathrm{GeV}$ (Fig. 29), using the fully $p_{T}$-integrated yield and $\left\langle p_{T}\right\rangle$ is expected to produce better results. Once such data become available, its effect on the posterior distribution needs to be verified.

\section{D. $\sqrt{s_{N N}}=39 \mathrm{GeV}$}

The experimental data available at $\sqrt{s_{N N}}=39 \mathrm{GeV}$ are a subset of that at $19.6 \mathrm{GeV}$; information about the pseudorapidity dependence of charged-particle yields is not available.
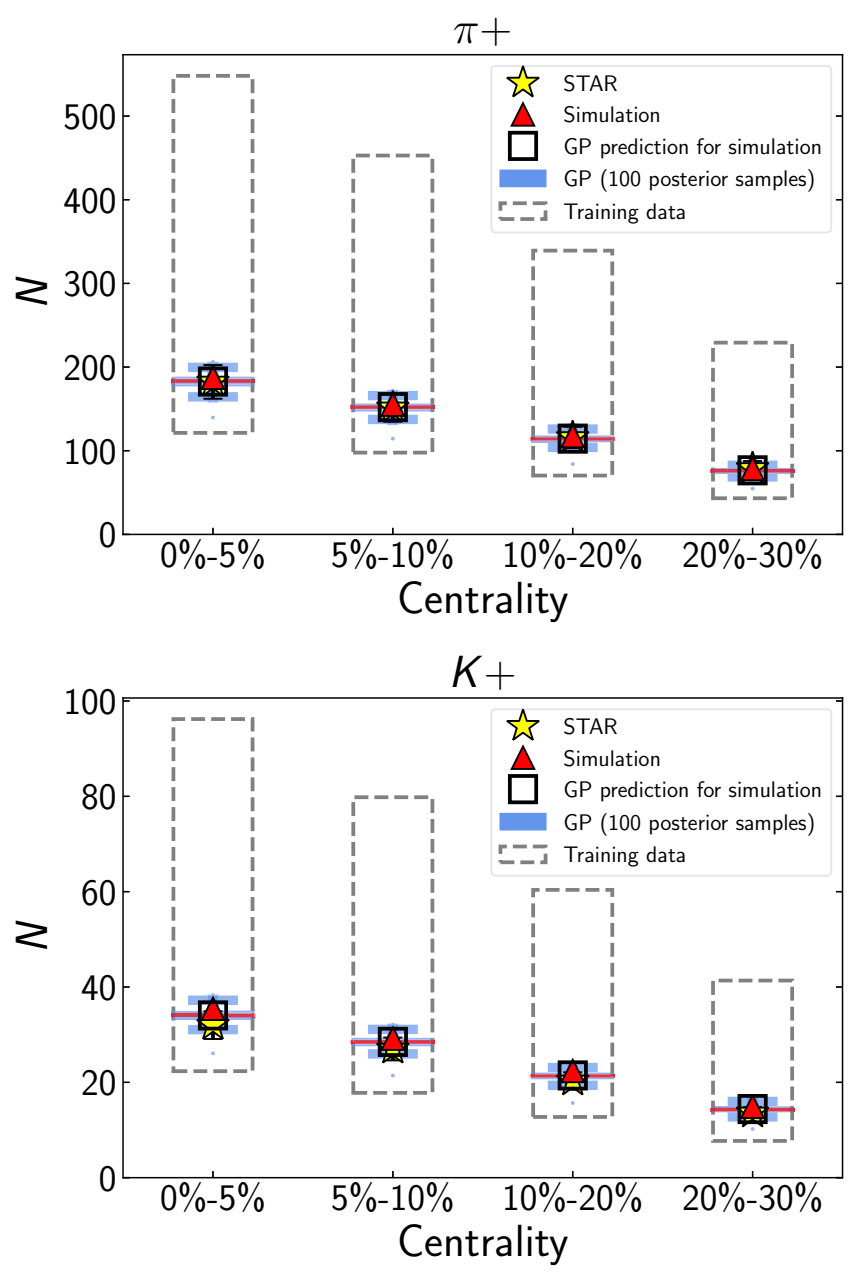

FIG. 13. Centrality dependence of positive pion and kaon yields at midrapidity at $\sqrt{s_{N N}}=39 \mathrm{GeV}$. Experimental data from Ref. [12].

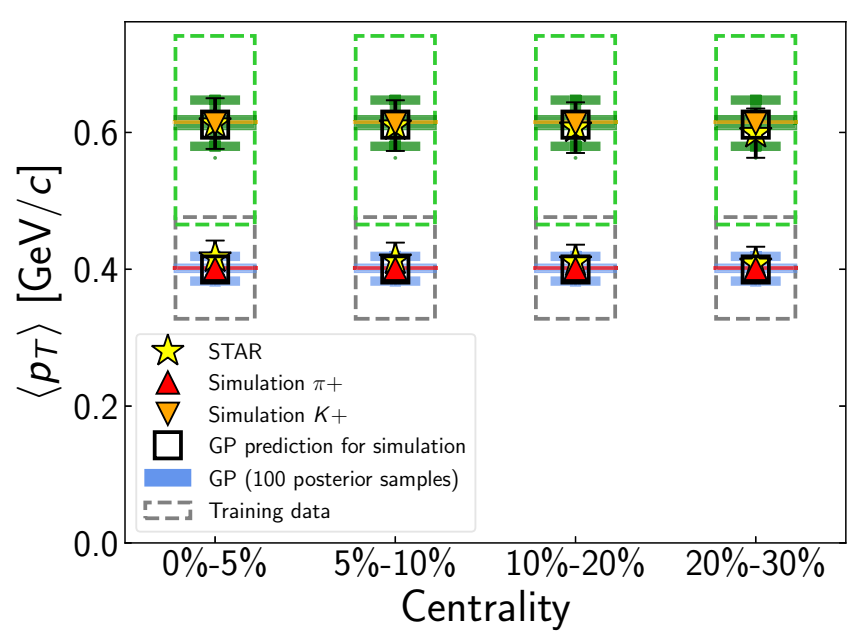

FIG. 14. Centrality dependence of mean transverse momentum of $\pi^{+}$(red up triangles) and $K^{+}$(orange down triangles) at $\sqrt{s_{N N}}=$ $39 \mathrm{GeV}$. Experimental data from Ref. [12].

As the earliest possible starting time remains favored (see Fig. 12), the $\tau_{0}$ distribution shifts below $1.0 \mathrm{fm}$, and the other distributions also peak at lower values, in particular, the switching energy density $\epsilon_{\mathrm{Sw}}$.

Both $\tau_{0}$ and $W_{\text {long }}$ are strongly constrained and we thus do not observe any correlations between these initial-state parameters and the effective shear viscosity at this energy. In addition, as at $\sqrt{s_{N N}}=19.6 \mathrm{GeV}$, no visible correlation is observed between $\eta / s$ and $W_{\text {trans }}$.

However, due to the lower peak value, the switching energy density $\epsilon_{\mathrm{SW}}$ is no longer artificially constrained by the prior, and it becomes apparent that the shear viscosity and the switching energy density have a positive correlation. At the same time, $W_{\text {trans }}$ is anticorrelated with $\epsilon_{\mathrm{SW}}$. Running the hydrodynamic model longer with a smaller viscosity would lead to an increase in the elliptic flow; this is compensated by smearing the initial density fluctuations in the transverse plane. So while

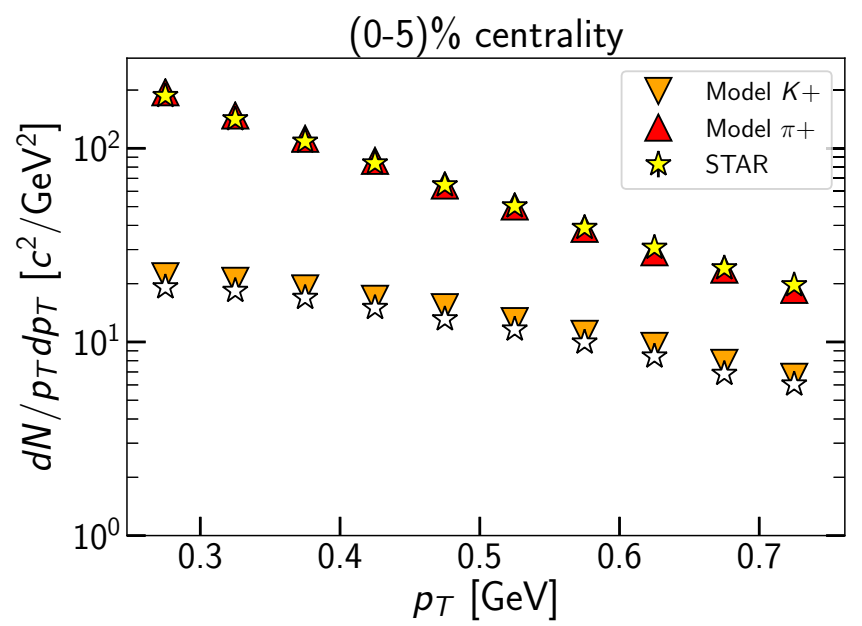

FIG. 15. Transverse momentum of $\pi^{+}$(red up triangles) and $K^{+}$ (orange down triangles) for $(0-5) \%$ centrality at $\sqrt{s_{N N}}=39 \mathrm{GeV}$. Experimental data from Ref. [12]. 


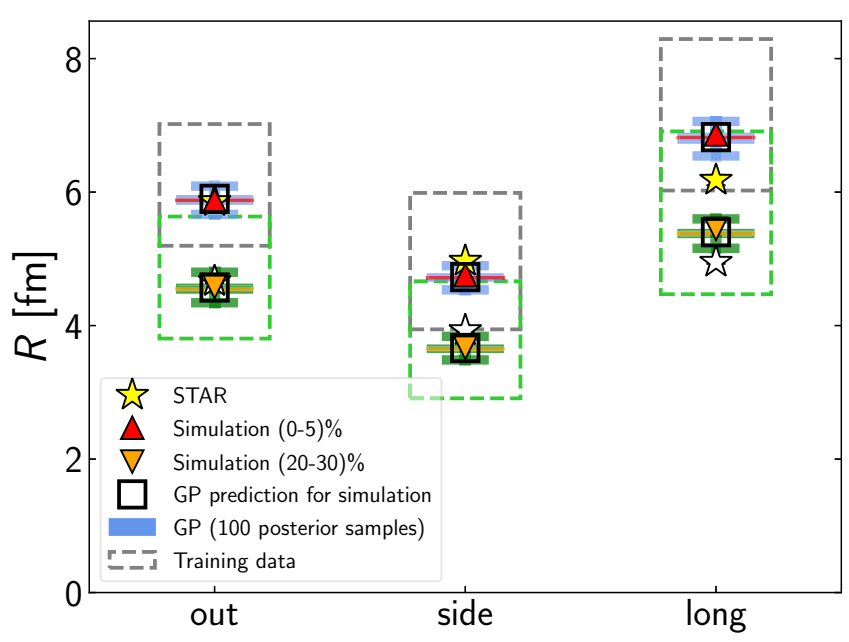

FIG. 16. $R_{\text {out }}, R_{\text {side }}$, and $R_{\text {long }}$ of charged $\pi$ at $\left\langle k_{T}\right\rangle \approx 0.22 \mathrm{GeV} / c$ at $(0-5) \%$ and $(20-30) \%$ centralities (red up triangles and orange down triangles, respectively) at $\sqrt{s_{N N}}=39 \mathrm{GeV}$. Experimental data from Ref. [11].

there is no direct correlation between $\eta / s$ and $W_{\text {trans }}$, they are indirectly linked by their simultaneous adjustments with respect to $\epsilon_{\mathrm{SW}}$. This interplay between multiple parameters illustrates the importance of simultaneously calibrating all the parameters.

The results for the actual observables are very similar to $\sqrt{s_{N N}}=19.6 \mathrm{GeV}$. Pion and kaon yields are again well reproduced (Fig. 13), as is their mean $p_{T}$ (Fig. 14), resulting in a good agreement with the measured transverse momentum spectra (Fig. 15).

Regarding the HBT radii (Figs. 16 and 17), $R_{\text {out }}$ is still well described with peak parameter values. However, in addition to the underestimation of $R_{\text {side }}$, we find $R_{\text {long }}$ to be overestimated at this energy. As $R_{\text {long }}$ has been associated with the freeze-out time and and temperature of the system [34] and should also be sensitive to the shear viscosity [35], increasing the weight

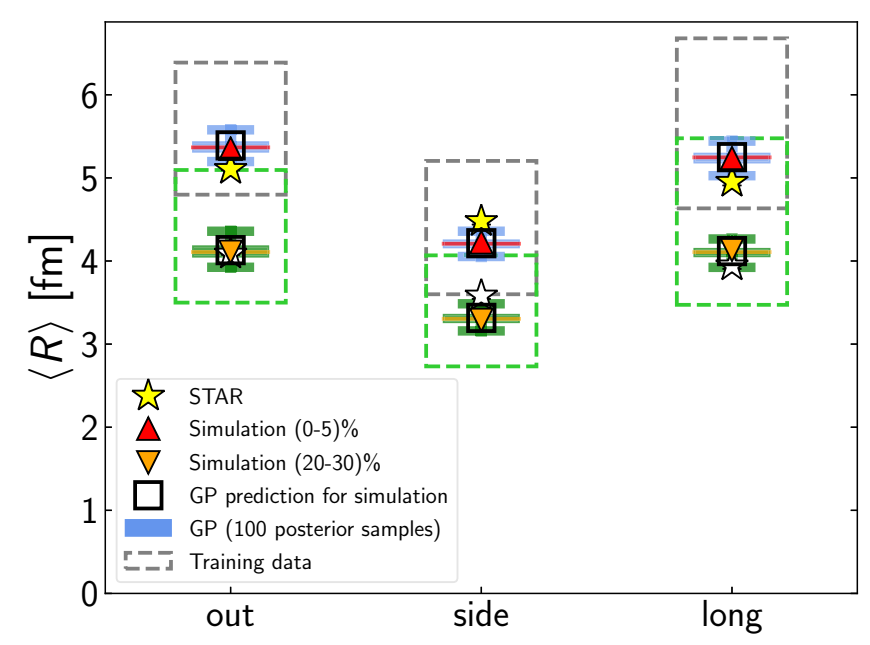

FIG. 17. $R_{\text {out }}, R_{\text {side }}$, and $R_{\text {long }}$ of charged $\pi$ averaged over $0.15 \mathrm{GeV} / c<k_{T}<0.6 \mathrm{GeV} / c$ at $(0-5) \%$ and (20-30)\% centrality at $\sqrt{s_{N N}}=39 \mathrm{GeV}$. Experimental data from Ref. [11].

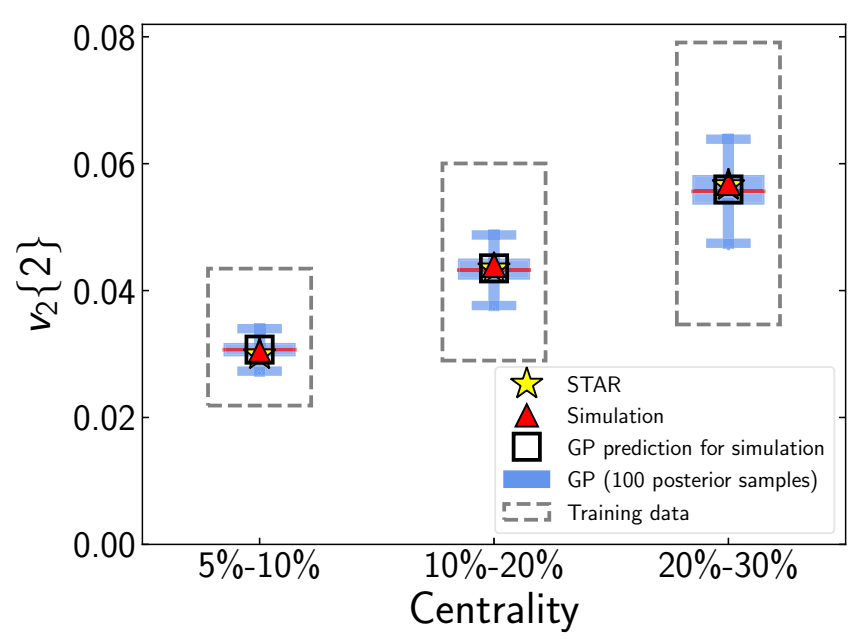

FIG. 18. Centrality dependence of charged particle $v_{2}\{2\}$ at $\sqrt{s_{N N}}=39 \mathrm{GeV}$. Experimental data from Ref. [32].

of HBT observables at this energy might have an effect on the most probable values of $\eta / s$ and $\epsilon_{\mathrm{SW}}$.

The results for $v_{2}\{2\}$ (Fig. 18) and $\Omega$ (Fig. 19) support the conclusions drawn from $\sqrt{s_{N N}}=19.6 \mathrm{GeV}$ results. Here the agreement with experimental data is good also for $(20-30) \%$ centrality, as the initial allowed hydro starting time is lower and likely appropriate for a wider range of centralities.

The transverse momentum spectrum of $\Omega$ is in better agreement with the STAR data at $\sqrt{s_{N N}}=39 \mathrm{GeV}$ compared to $\sqrt{s_{N N}}=19.6 \mathrm{GeV}$. The total yield is still overestimated, however.

\section{E. $\sqrt{s_{N N}}=62.4 \mathrm{GeV}$}

The highest collision energy investigated in this analysis has also the most comprehensive set of observables available, including information about the $p_{T}$ spectra of identified particles for several centralities. Instead of calibrating the model on the yield of pions and kaons separately, the $K^{+} / \pi^{+}$ratio

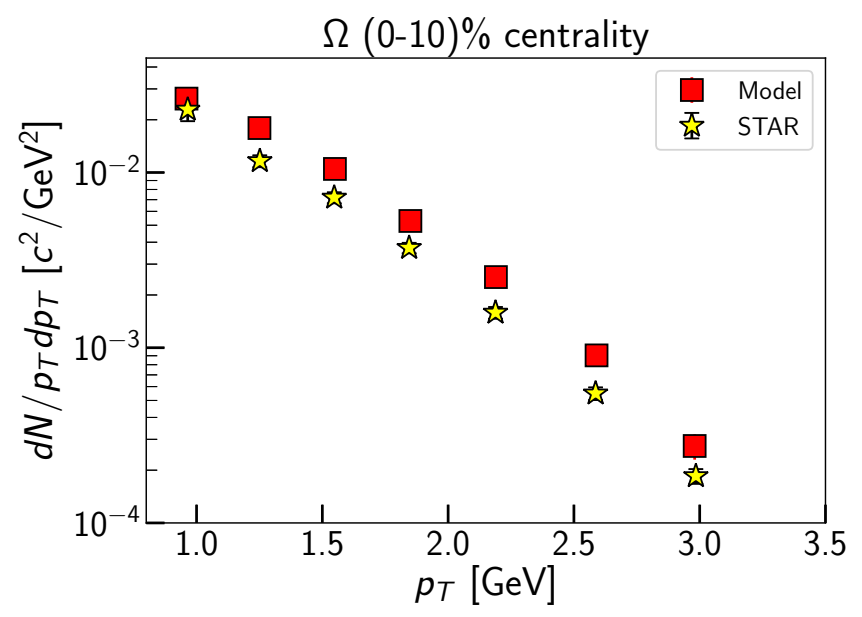

FIG. 19. Transverse momentum spectrum of $\Omega$ at $\sqrt{s_{N N}}=$ $39 \mathrm{GeV}$. Experimental data from Ref. [33]. 


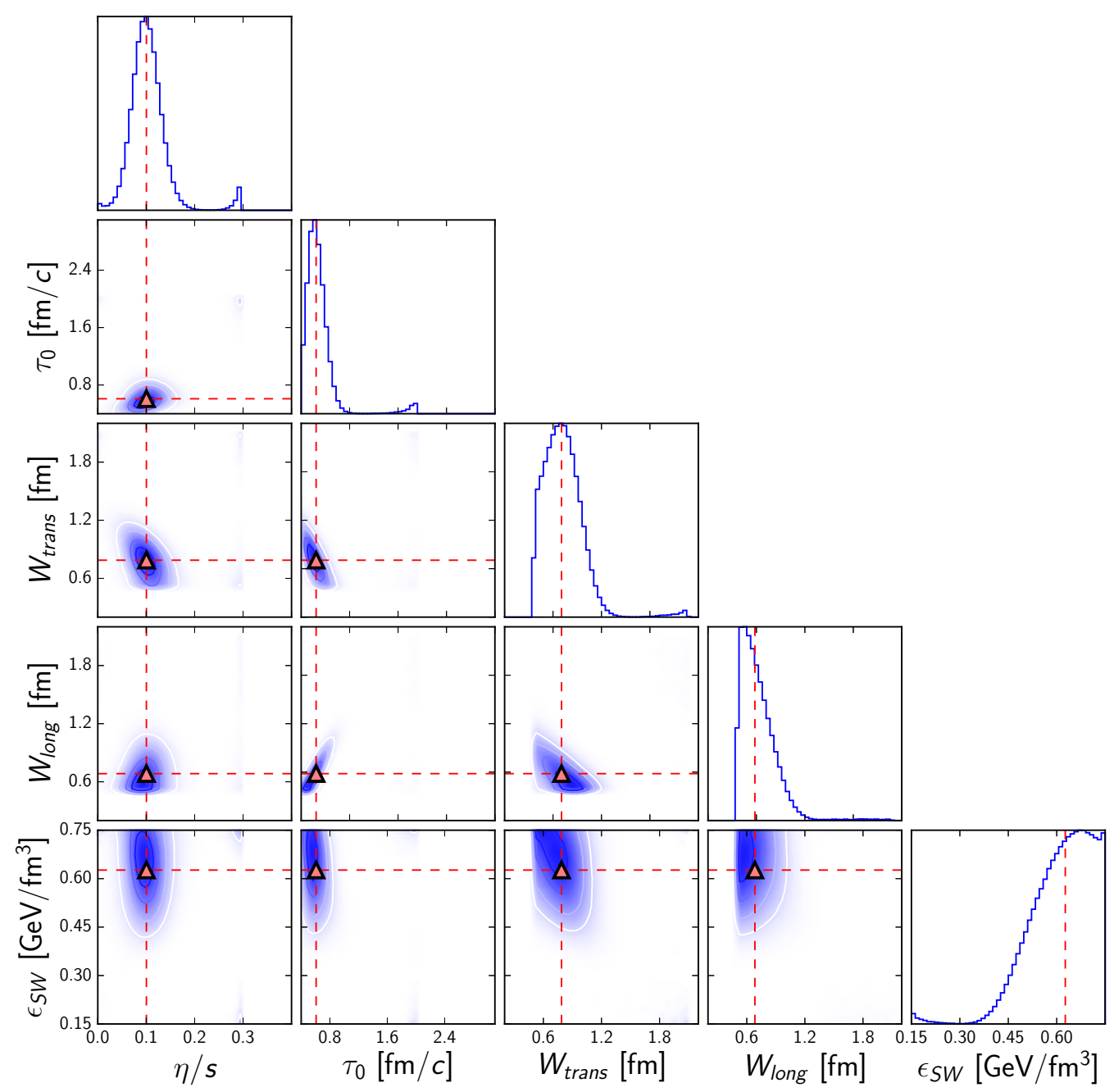

FIG. 20. Representation of the posterior probability distribution of the input parameters at $\sqrt{s_{N N}}=62.4 \mathrm{GeV}$. See caption of Fig. 2 for details.

was used instead. As the majority of charged particles consists of pions, calibrating the model on the centrality dependence of $N_{\text {ch }}$ was deemed sufficient to constrain the total number of pions when combined with the kaon/pion ratio.

The posterior probability distribution for $62.4 \mathrm{GeV}$, shown in Fig. 20, shows similar trends as the distributions at the lower energies. Early starting time for the hydrodynamic evolution is preferred, together with a longitudinally narrow Gaussian smoothing for the initial density. The transverse width of the smearing functions has narrowed further, leading to $W_{\text {trans }}$ and $W_{\text {long }}$ being roughly equivalent for this energy. The effective shear viscosity over entropy density ratio has a clear peak at 0.1 ; overall, the posterior probability distribution of $\eta / s$ changes very gradually over the investigated beam energies. Like at $19.6 \mathrm{GeV}$, and unlike at $39 \mathrm{GeV}$, a high value of the switching energy density is preferred.

At this highest investigated collision energy, we see a negative correlation between the shear viscosity and the transverse smearing factor, which has been expected based on flowbased arguments. It can be speculated that running the initial hadron transport beyond a time of $0.8 \mathrm{fm} / c$ already smears the initial state to the extent that the importance of initial-state fluctuations on elliptic flow is diminished at lower collision energies. On the other hand, there is no clear correlation between $\eta / s$ and $\epsilon_{\mathrm{SW}}$ at this highest investigated collision energy.

As illustrated in Fig. 21, the simulation using the peak parameter values gives a good description of the centrality dependence of charged particle multiplicity at midrapidity, especially considering the wide range of $N_{\mathrm{ch}}$ in the prior range.

The full charged particle pseudorapidity distributions for $(0-3) \%$ and $(20-25) \%$ centralities at $\sqrt{s_{N N}}=62.4 \mathrm{GeV}$ are shown in Fig. 22. The systematic difference between emulator prediction and actual simulation, observed at $\sqrt{s_{N N}}=$ $19.6 \mathrm{GeV}$, is present also at this higher collision energy. However, here the emulator prediction is systematically overestimating the midrapidity yield, in contrast to the systematic underestimation seen at $19.6 \mathrm{GeV}$.

The simulations run with median values agree with PHOBOS data overall quite well at (0-3)\% centrality. At (20-25)\% centrality, the charged particle yields are somewhat below the data both at $\eta \approx 0$ and again at $|\eta|>2.0$. 


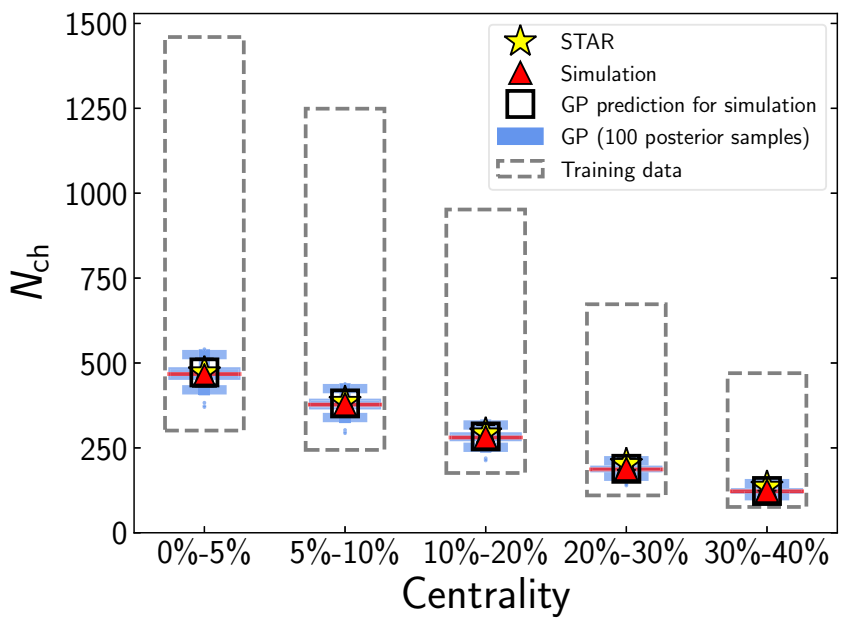

FIG. 21. Centrality dependence of charged particle multiplicity in $|\eta|<0.5$ at $\sqrt{s_{N N}}=62.4 \mathrm{GeV}$. Experimental data from STAR [30].
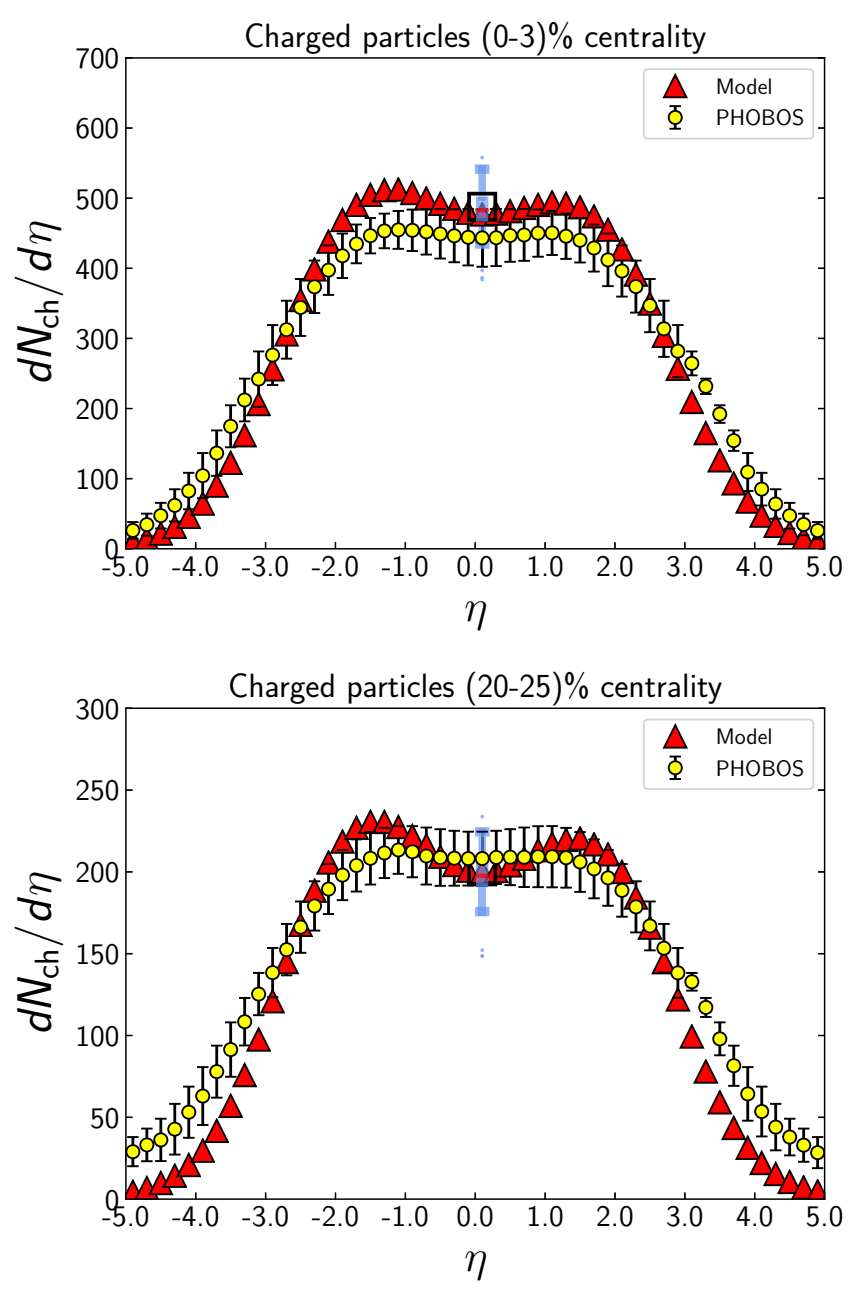

FIG. 22. Charged particle pseudorapidity distribution at $\sqrt{s_{N N}}=$ $62.4 \mathrm{GeV}$ for $(0-3) \%$ centrality (top) and $(20-25) \%$ centrality (bottom). PHOBOS data from Ref. [28].

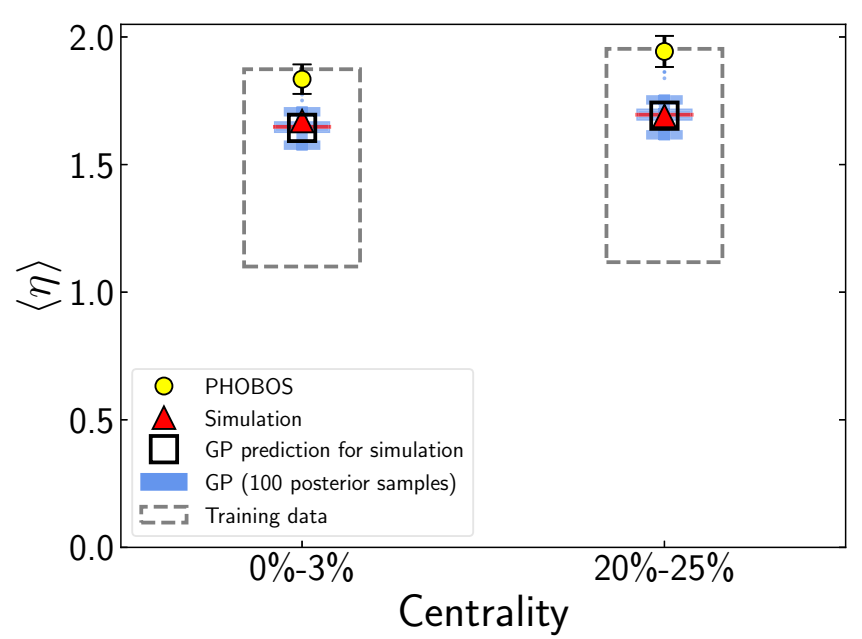

FIG. 23. Centrality dependence of average charged particle pseudorapidity $\langle\eta\rangle$ for $\eta>0.0$ at $\sqrt{s_{N N}}=62.4 \mathrm{GeV}$. Experimental values are estimated from PHOBOS data [28].

The mean pseudorapidities remain below the measurement, as shown in Fig. 23. At this energy, it appears possible to match PHOBOS results within the parameter prior. Based on the shape of $d N_{\mathrm{ch}} / d \eta$ from Fig. 22, matching $\langle\eta\rangle$ is likely to lead to a notable depletion of charged particles at $\eta \approx 0$, however.

Figure 24 shows that there is some tendency for the model to overestimate the abundance of kaons over pions. Overall, there is very little room variation even in the prior range of the particle ratio, so the shape of posterior will have little effect on this observable in any case. Despite the observed slight excess of kaons compared to pions, the mean transverse momenta of $\pi^{-}$and $K^{+}$is reproduced very well with the median parameter values and the $p_{T}$ spectra for $\pi^{-}, K^{+}$agrees well with PHOBOS results, as shown in Figs. 25 and 26.

The HBT analysis results presented in Figs. 27 and 28 are similar to the results at lower energies, in particular the results

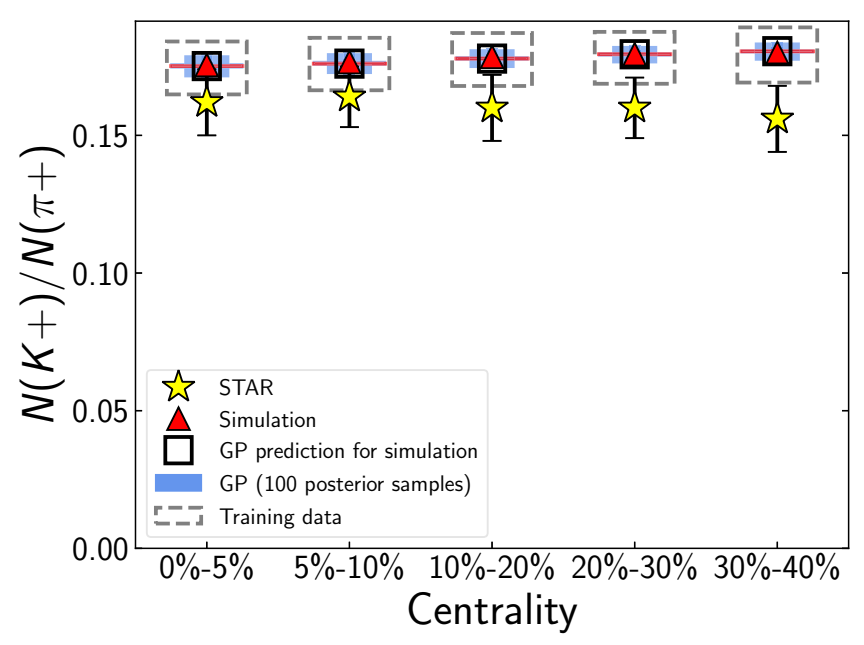

FIG. 24. Centrality dependence of kaon/pion ratio at $\sqrt{s_{N N}}=$ 62.4 GeV. Experimental data from STAR [30]. 


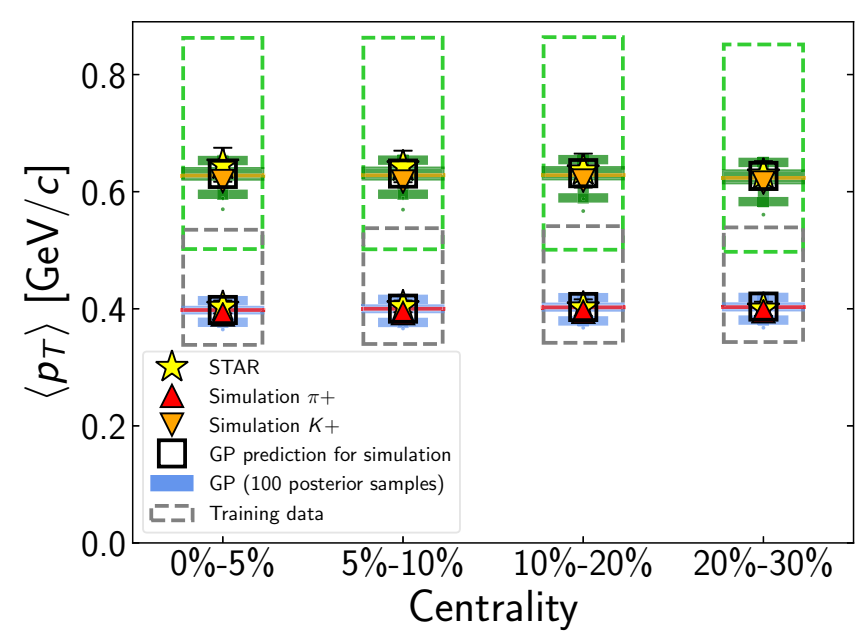

FIG. 25. The centrality dependence of mean transverse momentum of $\pi^{-}$(red up triangles) and $K^{+}$(orange down triangles) at $\sqrt{s_{N N}}=62.4 \mathrm{GeV}$. STAR data from Ref. [12].

at $\sqrt{s_{N N}}=39 \mathrm{GeV}: R_{\text {out }}$ is described well, while $R_{\text {long }}$ values from model output are systematically too large compared to data, while $R_{\text {side }}$ is notably smaller than the value determined from measurements. The fact that the behavior of HBT radii at 39 and $62.4 \mathrm{GeV}$ is very similar, despite the large difference in the applied value of the switching energy density, suggests that $R_{\text {long }}$ is more sensitive to $\eta / s$ than $\epsilon_{\mathrm{SW}}$.

Finally, we show the transverse momentum spectra of $\Omega$ and proton in Fig. 29. In contrast to the results at lower collision energies, the fully $p_{T}$-integrated $\Omega$ multiplicity and mean transverse momentum were used in the data calibration, leading to a good agreement with measured $p_{T}$ spectra; the total multiplicity is slightly overestimated. Also the proton spectrum, which was not part of the data calibration, matches quite well with the PHOBOS data, although slight overabundance of protons is evident from the figure.

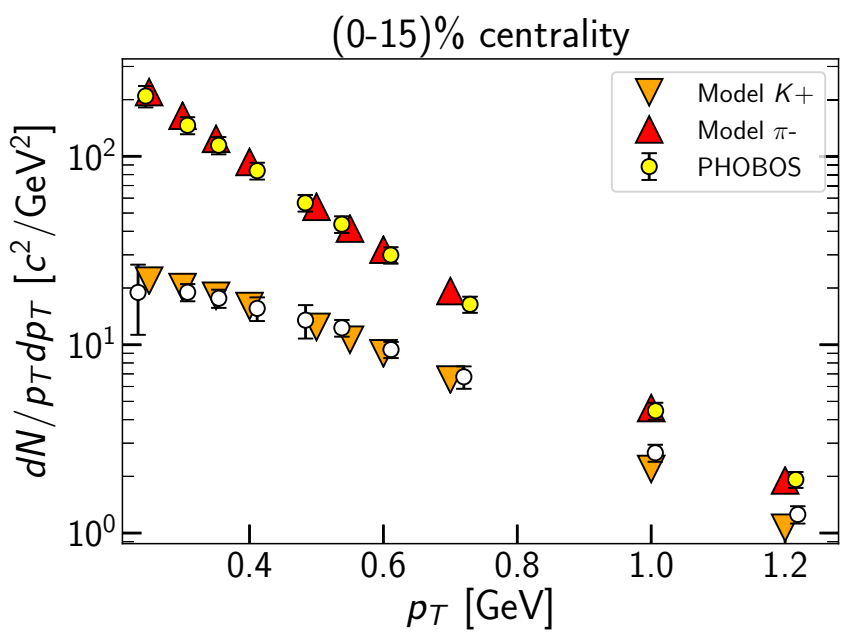

FIG. 26. The transverse momentum distributions of $\pi^{-}$(red up triangles) and $K^{+}$(orange down triangles) for (0-15)\% centrality at $\sqrt{s_{N N}}=62.4 \mathrm{GeV}$. PHOBOS data from Ref. [36].

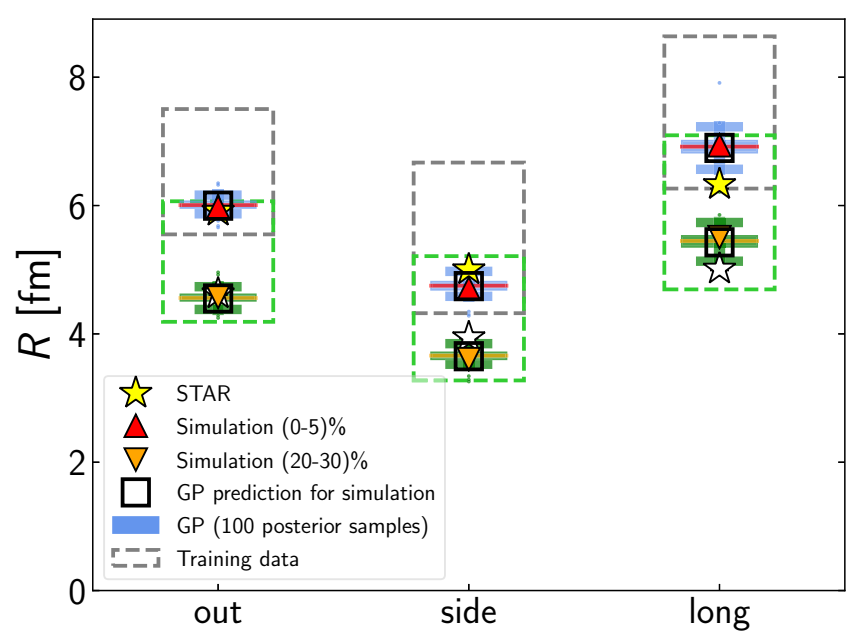

FIG. 27. $R_{\text {out }}, R_{\text {side }}$, and $R_{\text {long }}$ of charged $\pi$ at $\left\langle k_{T}\right\rangle \approx 0.22 \mathrm{GeV} / c$ at (0-5)\% and (20-30)\% centrality (red up triangles and orange down triangles, respectively) at $\sqrt{s_{N N}}=62.4 \mathrm{GeV}$. Experimental data from Ref. [11].

\section{F. Collision energy dependence of the Bayesian posteriors}

The collision energy dependence of the system properties is illustrated in Figs. 30-34. The symbols indicate the median values, while the error bars represent the $90 \%$ confidence range (i.e., smallest $5 \%$ and largest $5 \%$ of the parameter values from the posterior distribution are excluded).

The present analysis finds the effective $\eta / s$ during the hydrodynamical stage of the system evolution to have a modest dependence on collision energy, with median value decreasing from 0.176 at $\sqrt{s_{N N}}=19.6 \mathrm{GeV}$ to 0.100 at $\sqrt{s_{N N}}=$ 62.4 GeV (see Fig. 30). However, as the uncertainties increase toward lower values of $\sqrt{s_{N N}}$, a possibility for a constant value of $\eta / s \approx 0.10-0.15$ cannot be currently excluded.

One might argue that the observed dependence on $\sqrt{s_{N N}}$ could be explained by introducing a temperature dependence.

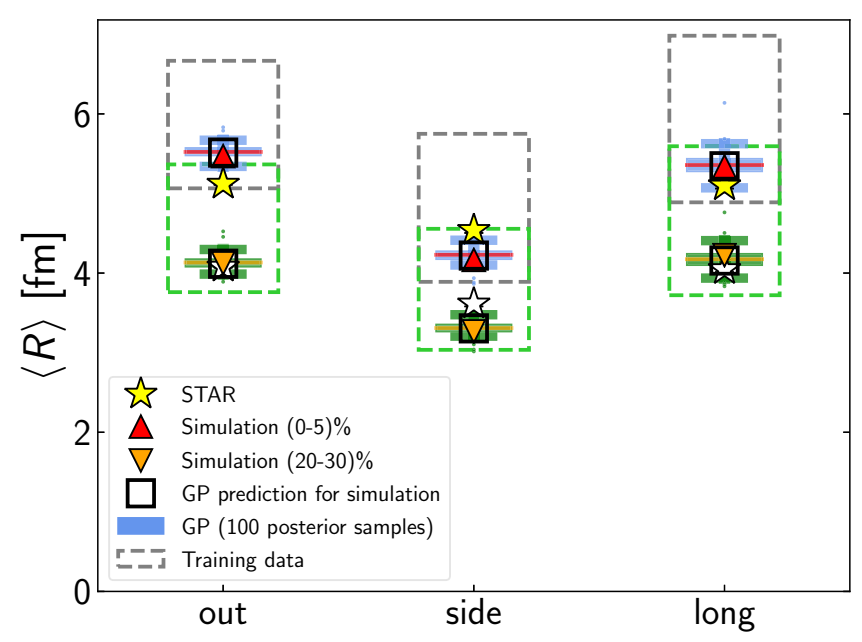

FIG. 28. $R_{\text {out }}, R_{\text {side }}$, and $R_{\text {long }}$ of charged $\pi$ averaged over $0.15 \mathrm{GeV} / c<k_{T}<0.6 \mathrm{GeV} / c$ at $(0-5) \%$ and (20-30)\% centralities at $\sqrt{s_{N N}}=62.4 \mathrm{GeV}$. Experimental data from Ref. [11]. 

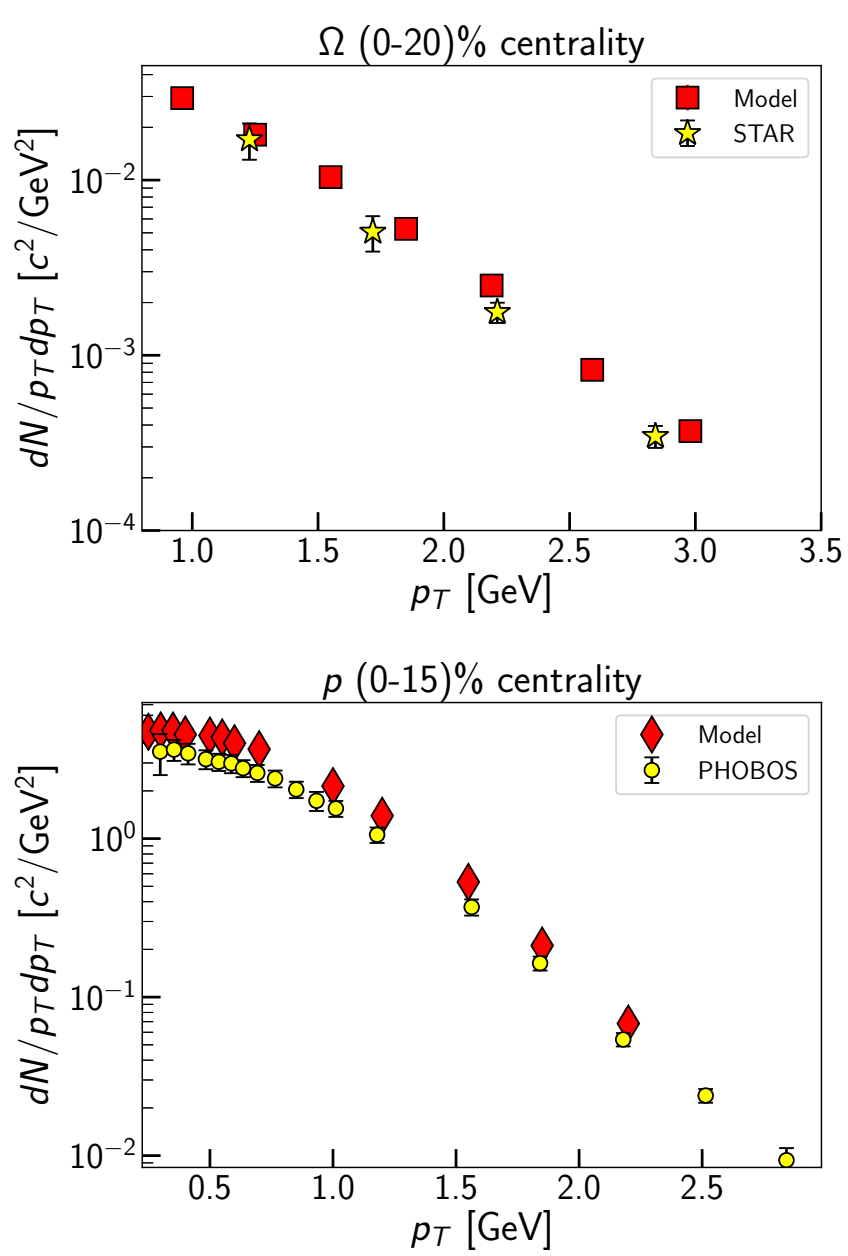

FIG. 29. Transverse momentum spectrum of $\Omega$ (top) and proton (bottom) at $\sqrt{s_{N N}}=62.4 \mathrm{GeV}$. Note that proton data were not used in the data calibration process. STAR data from Ref. [37] and PHOBOS data from Ref. [36].

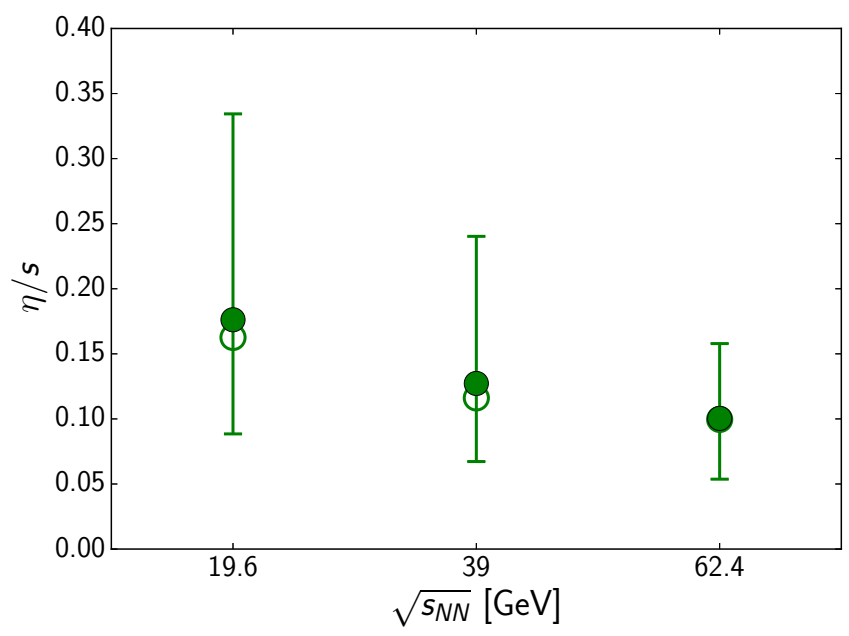

FIG. 30. Collision energy dependence of the effective shear viscosity over entropy density ratio $\eta / s$ during the hydrodynamical evolution. Error bars represent $90 \%$ confidence range around the median value (filled circles). Open symbols indicate the peak position of the distribution.

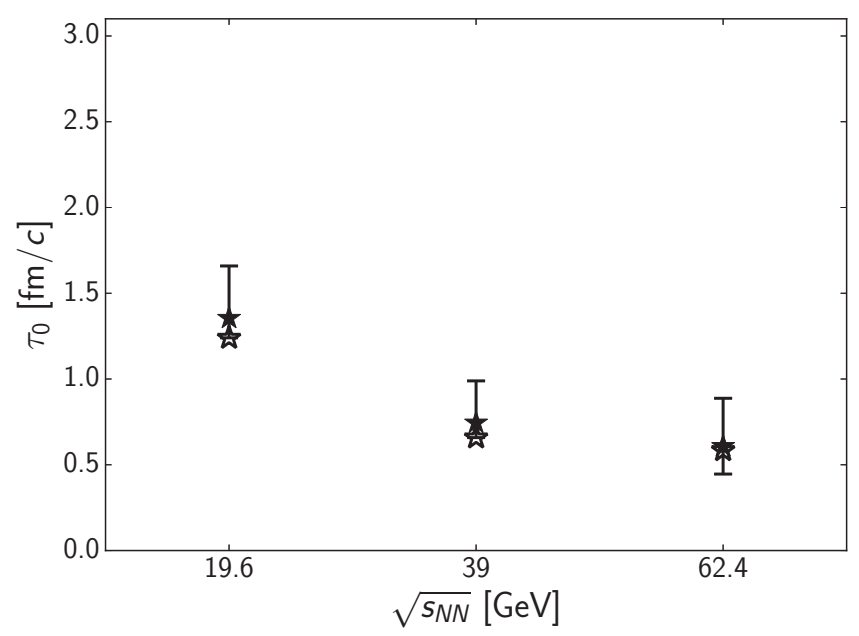

FIG. 31. Collision energy dependence of the hydro starting time $\tau_{0}$. Error bars represent $90 \%$ confidence range around the median value (filled stars). Open symbols indicate the peak position of the distribution.

However, since the studies performed at LHC energies suggest that $\eta / s(T)$ in the QGP increases as a function of temperature, whereas the peak temperatures in our study will decrease as a function of decreasing collision energies, the observed trend in the effective $\eta / s$ seems to oppose the expectation from a purely temperature-dependent shear viscosity.

Regarding the three parameters controlling the initial state for the hydrodynamic model, the transport-to-hydro switching time parameter $\tau_{0}$ is found to favor the earliest possible starting times for the hydrodynamical evolution, between 0.5 and $1.0 \mathrm{fm} / c$. The increase to $1.36 \mathrm{fm} / c$ for $\sqrt{s_{N N}}=$ $19.6 \mathrm{GeV}$ is induced by the increase in the earliest possible starting time at lower energies, due to the longer time it takes for the two nuclei to interpenetrate each other.

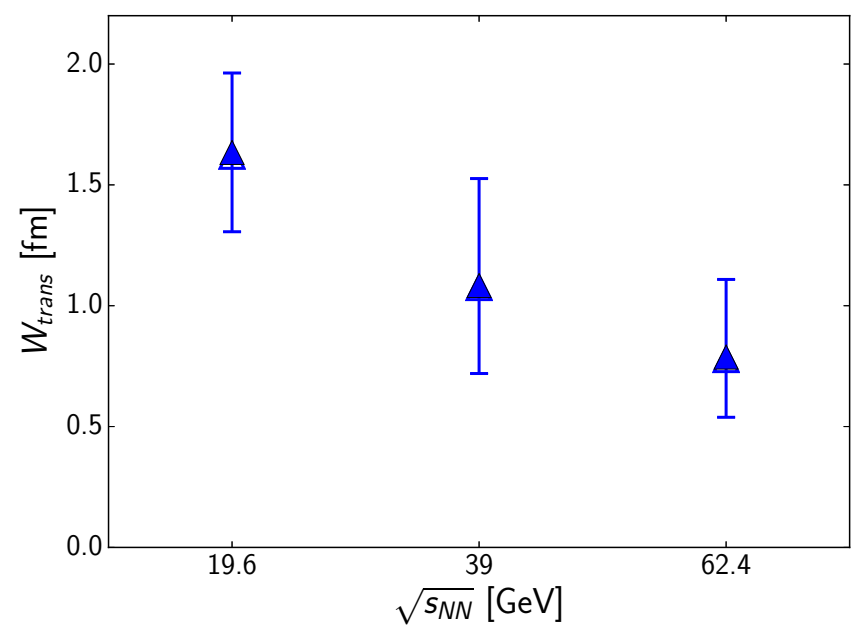

FIG. 32. Collision energy dependence of the smearing factor $W_{\text {trans }}$. Error bars represent $90 \%$ confidence range around the median value (filled triangles). Open symbols indicate the peak position of the distribution. 


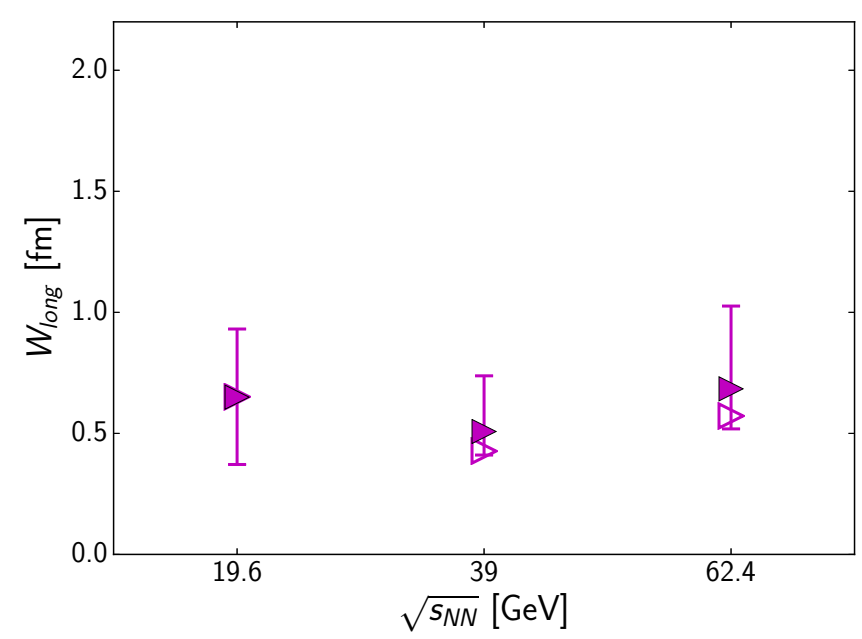

FIG. 33. Collision energy dependence of the smearing factor $W_{\text {long }}$. Error bars represent $90 \%$ confidence range around the median value (filled triangles). Open symbols indicate the peak position of the distribution.

The transverse width of the Gaussian representation of initial particles $W_{\text {trans }}$ shows a strong collision energy dependence, with the most probable value of $W_{\text {trans }} \approx 1.6 \mathrm{fm}$ at $\sqrt{s_{N N}}=$ $19.6 \mathrm{GeV}$ decreasing to $W_{\text {trans }} \approx 0.8 \mathrm{fm}$ at $\sqrt{s_{N N}}=62.4 \mathrm{GeV}$. On the other hand, the longitudinal smearing value is found to be roughly constant, $W_{\text {long }} \approx 0.5-0.7 \mathrm{fm}$.

The particlization energy density $\epsilon_{\mathrm{SW}}$ (the transition from hydrodynamics back to a hadron transport afterburner) has a notably different probability distribution at $\sqrt{s_{N N}}=39 \mathrm{GeV}$ compared to $\sqrt{s_{N N}}=19.6$ and $62.4 \mathrm{GeV}$. As the charged particle pseudorapidity distribution is included in the analysis for both $\sqrt{s_{N N}}=19.6$ and $62.4 \mathrm{GeV}$, but is not available for $\sqrt{s_{N N}}=39 \mathrm{GeV}, d N_{\mathrm{ch}} / d \eta$ would seem a likely cause for the discrepancy. However, as seen in Fig. 35, excluding the charged

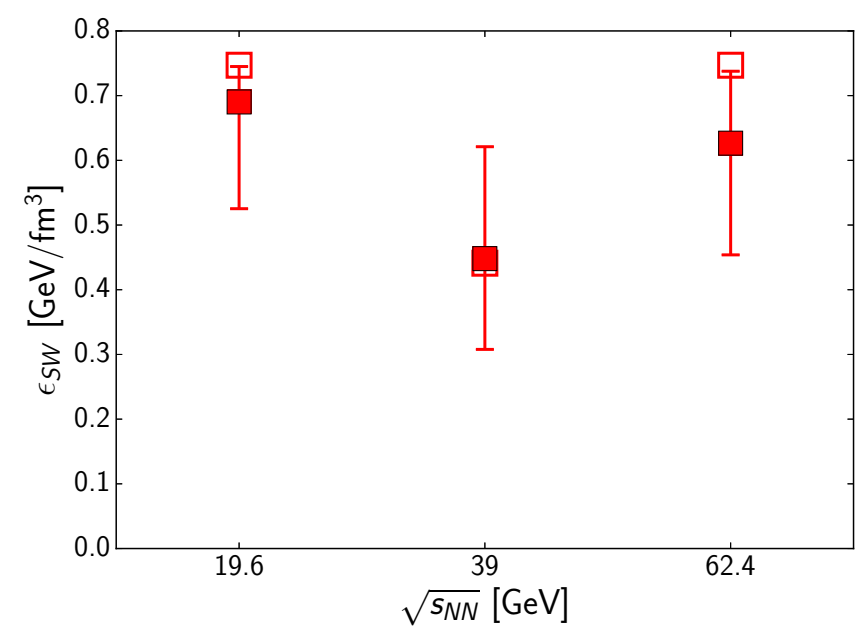

FIG. 34. Collision energy dependence of the switching energy density $\epsilon_{\mathrm{SW}}$. Error bars represent $90 \%$ confidence range around the median value (filled squares). Open symbols indicate the peak position of the distribution.

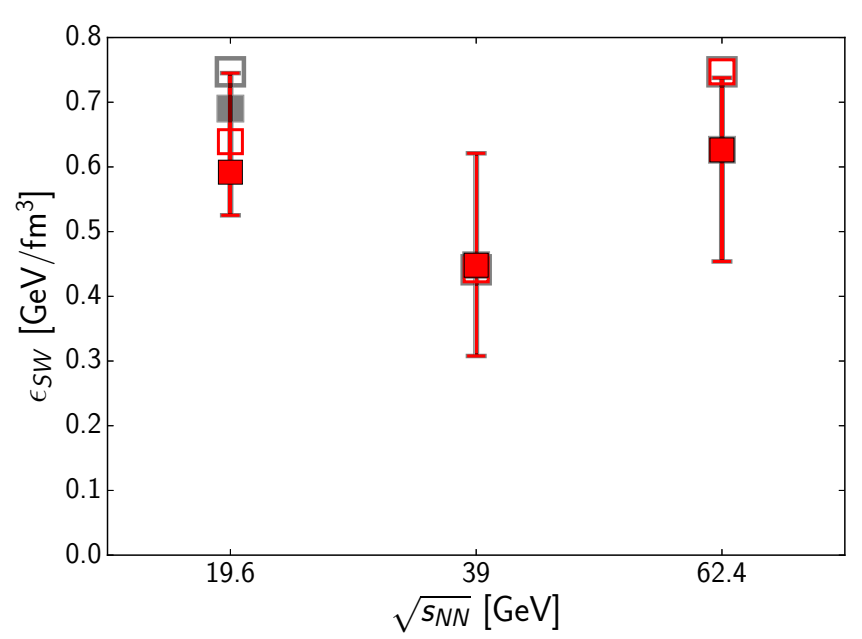

FIG. 35. Collision energy dependence of the switching energy density $\epsilon_{\mathrm{SW}}$, if charged particle pseudorapidity distribution is excluded from the Bayesian analysis (red), compared to the result from full analysis (in gray).

particle pseudorapidity distribution (i.e., both $d N_{\text {ch }} /\left.d \eta\right|_{\eta=0.1}$ and $\langle\eta\rangle)$ has a visible effect only on the posterior distribution of $\epsilon_{\mathrm{SW}}$ at $\sqrt{s_{N N}}=19.6 \mathrm{GeV}$, and even there the change is within $90 \%$ confidence range, which remains unaffected. As the effect is even smaller on the posterior distributions of the other parameters, this assures us that our analysis is indeed robust and not overly sensitive to any single observable.

\section{SUMMARY}

Using state-of-the-art statistical methods, we have determined the highest likelihood input parameters and related uncertainties of the state-of-the-art (3+1)-D viscous hydrodynamics + transport hybrid model for three collision energies $\sqrt{s_{N N}}=19.6,39$, and $62.4 \mathrm{GeV}$, simultaneously fitting all five model parameters to a comprehensive set of experimental data for each energy.

We find a moderate collision energy dependence for the most likely value of the effective shear viscosity over entropy density ratio in the QGP phase. However, as the uncertainties at lower energies are still considerable, it remains possible that the optimal value of $\eta / s$ during hydrodynamical evolution is a constant over the investigated beam energy range, with a value between 0.10 and 0.15 .

The best-fit value of transverse smearing parameter $W_{\text {trans }}$ is also found to depend on collision energy, with larger values at lower collision energies, while $W_{\text {long }}$ remains almost constant with respect to $\sqrt{s_{N N}}$. As the collision energy dependence of these smearing parameters lack an obvious physical motivation, it becomes necessary to impose more stringent theoretical constraints on the initial state for the future studies.

We find that the fluidization - the transition from a transport description of the system to hydrodynamics-is likely to happen very early, and the observed collision energy dependence mainly stems from the constraint on the minimum time, Eq. (20). 
For the hydro-to-transport switching energy density $\epsilon_{\mathrm{SW}}$, an early switch from hydrodynamics to hadron transport is preferred for $\sqrt{s_{N N}}=19.6$ and $62.4 \mathrm{GeV}$, while a lower value is found to be more probable for $\sqrt{s_{N N}}=39 \mathrm{GeV}$. However, in all cases $\epsilon_{\mathrm{SW}}>0.3 \mathrm{GeV} / \mathrm{fm}^{3}$, i.e., high enough to justify the assumption of a chemical equilibrium at particlization. It remains to be seen if future data are able to provide additional constraints on $\epsilon_{\mathrm{SW}}$.

While the values of the medium parameters extracted in the present analysis already provide a very good global fit on the beam energy scan data, there remains some room for improvement on pseudorapidity distribution and HBT radii, for example. The current model setup also ignores bulk viscosity and net-baryon diffusion; testing the latter also requires a solution for the proton feed-down correction problem currently hampering model-to-data comparisons on baryon observables in beam energy scan range. It is also important to keep in mind that the present analysis is limited to a specific initial state model, UrQMD. An important future study would be to quantify the uncertainties related to the choice of initial state.

Overall the median values from the statistical analysis largely validate the parameter estimates presented in Ref. [8]. However, as a crucial difference, we find the uncertainties related to the extracted effective shear viscosity to be severely underestimated in that previous study. This illustrates the importance of exploring the full input parameter space in model-to-data comparisons. Bayesian statistics and Gaussian process emulators provide us the necessary tools to perform such analysis in a robust manner.

\section{ACKNOWLEDGMENTS}

This work has been supported by NSF Grant No. NSF-ACI1550225 and by DOE Grant No. DE-FG02-05ER41367. I.K. acknowledges partial support by the University of Florence grant Fisica dei plasmi relativistici: teoria e applicazioni moderne. CPU time was provided by the Open Science Grid, supported by DOE and NSF. We thank the Institute for Nuclear Theory at the University of Washington for its hospitality and the Department of Energy for partial support during the completion of this work.

\section{APPENDIX A: EXPERIMENTAL DATA}

The observables used in the analysis are summarized in Tables I-III.

Mean transverse momentum of $\Omega$ at $\sqrt{s_{N N}}=19.6$ and $39 \mathrm{GeV}$ has been computed from published $p_{T}$ spectrum data. To avoid introducing additional uncertainties related to extrapolation of the spectra at low $p_{T}$, the mean is computed within the range of data points, $p_{T}=1-5 \mathrm{GeV} / c$.

$\left\langle R_{\text {out }}\right\rangle,\left\langle R_{\text {side }}\right\rangle$, and $\left\langle R_{\text {long }}\right\rangle$ refer to simple averages over the four $k_{T}$ bins, covering the range $0.15 \mathrm{GeV} / c<k_{T}<$ $0.6 \mathrm{GeV} / c$.

The data in Ref. [32] for event plane elliptic flow $v_{2}\{\mathrm{EP}\}$ at $\sqrt{s_{N N}}=62.4 \mathrm{GeV}$ are presented as a function of pseudorapidity $\eta$. To improve our accuracy on determining $v_{2}$, we have taken advantage of the fact that $v_{2}\{\mathrm{EP}\}$ is practically constant between $-0.3<\eta<0.3$ at this energy and compare the value
TABLE I. Calibration data at $\sqrt{s_{N N}}=19.6 \mathrm{GeV}$.

\begin{tabular}{lcc}
\hline \hline Observable & Centrality (\%) & Ref. \\
\hline$d N_{\text {ch }} / d \eta$ at $\eta=0.1$ & $0-6,6-15,15-25$ & {$[28]$} \\
$\langle\eta\rangle$ for $\eta>0.0$ & $0-6,6-15,15-25$ & {$[28]$} \\
$N\left(\pi^{+}\right), N\left(\pi^{-}\right)$ & $0-5,5-10,10-20,20-30$ & {$[12]$} \\
$N\left(K^{+}\right), N\left(K^{-}\right)$ & $0-5,5-10,10-20,20-30$ & {$[12]$} \\
$\left\langle p_{T}\right\rangle$ for $\pi^{+}, \pi^{-}, K^{+}, K^{-}$ & $0-5,5-10,10-20,20-30$ & {$[12]$} \\
Charged particle $v_{2}\{2\}$ in $|\eta|<0.5$ & $5-10,10-20,20-30$ & {$[32]$} \\
$d N(\Omega) / d p_{T}$ at $p_{T}=1.01 \mathrm{GeV} / c$ & $0-10$ & {$[33]$} \\
$\left\langle p_{T}\right\rangle$ for $\Omega$ at $p_{T}=1.0-5.0 \mathrm{GeV} / c$ & $0-10$ & {$[33]$} \\
$R_{\text {out }}, R_{\text {side }}, R_{\text {long }}$ of charged & $0-5,20-30$ & {$[11]$} \\
$\pi$ at $\left\langle k_{T}\right\rangle \approx 0.22 \mathrm{GeV} / c$ & & \\
$\left\langle R_{\text {out }}\right\rangle,\left\langle R_{\text {side }}\right\rangle,\left\langle R_{\text {long }}\right\rangle$ of charged $\pi$ & $0-5,20-30$ & {$[11]$} \\
\hline \hline
\end{tabular}

TABLE II. Calibration data at $\sqrt{s_{N N}}=39 \mathrm{GeV}$.

\begin{tabular}{lcr}
\hline \hline Observable & Centrality $(\%)$ & Ref. \\
\hline$N\left(\pi^{+}\right), N\left(\pi^{-}\right)$ & $0-5,5-10,10-20,20-30[12]$ \\
$N\left(K^{+}\right), N\left(K^{-}\right)$ & $0-5,5-10,10-20,20-30[12]$ \\
$\left\langle p_{T}\right\rangle$ for $\pi^{+}, \pi^{-}, K^{+}, K^{-}$ & $0-5,5-10,10-20,20-30[12]$ \\
$v_{2}\{2\}$ in $|\eta|<0.5$ & $5-10,10-20,20-30$ & {$[32]$} \\
$d N(\Omega) / d p_{T}$ at $p_{T}=0.96 \mathrm{GeV} / c$ & $0-10$ & {$[33]$} \\
$\left\langle p_{T}\right\rangle$ for $\Omega$ at $p_{T}=1.0-5.0 \mathrm{GeV} / c$ & $0-10$ & {$[33]$} \\
$R_{\text {out }}, R_{\text {side }}, R_{\text {long }}$ of charged & $0-5,20-30$ \\
$\pi$ at $\left\langle k_{T}\right\rangle \approx 0.22 \mathrm{GeV} / c$ & $0-5,20-30$ \\
$\left\langle R_{\text {out }}\right\rangle,\left\langle R_{\text {side }}\right\rangle,\left\langle R_{\text {long }}\right\rangle$ of charged $\pi$ & 0 \\
\hline \hline
\end{tabular}

TABLE III. Calibration data at $\sqrt{s_{N N}}=62.4 \mathrm{GeV}$.

\begin{tabular}{lcc}
\hline \hline Observable & Centrality (\%) & Ref. \\
\hline$N_{\text {ch }}$ in $|\eta|<0.5$ & $0-5,5-10,10-20,20-30$, & {$[30]$} \\
$d N_{\text {ch }} / d \eta$ at $\eta=0.1$ & $30-40$ & \\
$\langle\eta\rangle$ for $\eta>0.0$ & $0-3,20-25$ & {$[28]$} \\
$N\left(K^{+}\right) / N\left(\pi^{+}\right)$in $|\eta|<0.5$ & $0-5,5-10,10-20,20-30,30-40$ & {$[30]$} \\
$\left\langle p_{T}\right\rangle$ for $\pi^{-}, K^{+}$ & $0-5,10-20,20-30$ & {$[30]$} \\
$v_{2}\{\mathrm{EP}\}$ in $|\eta|<0.3$ & $10-40$ & {$[32]$} \\
$v_{2}\{$ corr $\}$ & $10-20,20-30,30-40$ & {$[38]$} \\
$N(\Omega)$ in $|y|<0.5$ & $0-20$ & {$[39]$} \\
$\left\langle p_{T}\right\rangle$ for $\Omega$ in $|y|<0.5$ & $0-20$ & {$[37]$} \\
$R_{\text {out }}, R_{\text {side }}, R_{\text {long }}$ of charged & $0-5,20-30$ & {$[11]$} \\
$\pi$ at $\left\langle k_{T}\right\rangle \approx 0.22$ GeV $/ c$ & & \\
$\left\langle R_{\text {out }}\right\rangle,\left\langle R_{\text {side }}\right\rangle,\left\langle R_{\text {long }}\right\rangle$ of & $0-5,20-30$ & \\
charged $\pi$ & & \\
\hline \hline
\end{tabular}

TABLE IV. Seven observables at $\sqrt{s_{N N}}=19.6 \mathrm{GeV}$ with most weight on principal component 0 ( $89.43 \%$ of total variance).

\begin{tabular}{ll}
\hline \hline Observable & Weight \\
\hline$N\left(K^{-}\right)(20-30) \%$ & 0.0634 \\
$N\left(K^{-}\right)(10-20) \%$ & 0.0576 \\
$N\left(\pi^{+}\right)(20-30) \%$ & 0.0568 \\
$N\left(K^{+}\right)(20-30) \%$ & 0.0566 \\
$N\left(\pi^{-}\right)(20-30) \%$ & 0.0548 \\
$N\left(K^{-}\right)(5-10) \%$ & 0.0534 \\
$d N_{\mathrm{ch}} / d \eta$ at $\eta=0.1(15-25) \%$ & 0.0516 \\
\hline \hline
\end{tabular}


TABLE V. Seven observables at $\sqrt{s_{N N}}=19.6 \mathrm{GeV}$ with most weight on principal component 1 (5.99\% of total variance).

\begin{tabular}{ll}
\hline \hline Observable & Weight \\
\hline$N(\Omega)(0-10) \%$ & 0.2759 \\
$\left\langle p_{T}\right\rangle\left(K^{-}\right)(20-30) \%$ & 0.0484 \\
$\left\langle p_{T}\right\rangle\left(K^{-}\right)(10-20) \%$ & 0.0451 \\
$\left\langle p_{T}\right\rangle\left(K^{-}\right)(5-10) \%$ & 0.0422 \\
$v_{2}\{2\}(20-30) \%$ & 0.0417 \\
$\left\langle p_{T}\right\rangle\left(K^{-}\right)(0-5) \%$ & 0.0401 \\
$\left\langle p_{T}\right\rangle\left(K^{+}\right)(20-30) \%$ & 0.0381 \\
\hline \hline
\end{tabular}

TABLE VI. Seven observables at $\sqrt{s_{N N}}=19.6 \mathrm{GeV}$ with most weight on principal component 2 (2.32\% of total variance).

\begin{tabular}{ll}
\hline \hline Observable & Weight \\
\hline$v_{2}\{2\}(20-30) \%$ & 0.3366 \\
$v_{2}\{2\}(10-20) \%$ & 0.2925 \\
$v_{2}\{2\}(5-10) \%$ & 0.2353 \\
$N(\Omega)(0-10) \%$ & 0.0376 \\
$\left\langle p_{T}\right\rangle\left(K^{+}\right)(0-5) \%$ & 0.0064 \\
$\left\langle p_{T}\right\rangle\left(K^{+}\right)(5-10) \%$ & 0.0058 \\
$\left\langle p_{T}\right\rangle\left(K^{-}\right)(0-5) \%$ & 0.0056 \\
\hline \hline
\end{tabular}

TABLE VII. Seven observables at $\sqrt{s_{N N}}=39 \mathrm{GeV}$ with most weight on principal component 0 ( $84.47 \%$ of total variance).

\begin{tabular}{ll}
\hline \hline Observable & Weight \\
\hline$N(\Omega)(0-10) \%$ & 0.1311 \\
$N\left(\pi^{-}\right)(20-30) \%$ & 0.0659 \\
$N\left(\pi^{+}\right)(20-30) \%$ & 0.0643 \\
$N\left(K^{-}\right)(20-30) \%$ & 0.0639 \\
$N\left(K^{-}\right)(10-20) \%$ & 0.0572 \\
$N\left(K^{+}\right)(20-30) \%$ & 0.0562 \\
$N\left(\pi^{-}\right)(10-20) \%$ & 0.0547 \\
\hline \hline
\end{tabular}

TABLE VIII. Seven observables at $\sqrt{s_{N N}}=39 \mathrm{GeV}$ with most weight on principal component 1 (9.37\% of total variance).

\begin{tabular}{ll}
\hline \hline Observable & Weight \\
\hline$N(\Omega)(0-10) \%$ & 0.4506 \\
$v_{2}\{2\}(20-30) \%$ & 0.0389 \\
$v_{2}\{2\}(10-20) \%$ & 0.0291 \\
$\left\langle p_{T}\right\rangle\left(K^{-}\right)(20-30) \%$ & 0.0282 \\
$\left\langle p_{T}\right\rangle\left(K^{-}\right)(10-20) \%$ & 0.0273 \\
$\left\langle p_{T}\right\rangle\left(K^{-}\right)(5-10) \%$ & 0.0272 \\
$\left\langle p_{T}\right\rangle\left(K^{-}\right)(0-5) \%$ & 0.0264 \\
\hline \hline
\end{tabular}

TABLE IX. Seven observables at $\sqrt{s_{N N}}=39 \mathrm{GeV}$ with most weight on principal component 2 (3.36\% of total variance).

\begin{tabular}{ll}
\hline \hline Observable & Weight \\
\hline$N(\Omega)(0-10) \%$ & 0.3287 \\
$v_{2}\{2\}(20-30) \%$ & 0.1671 \\
$v_{2}\{2\}(10-20) \%$ & 0.1255 \\
$v_{2}\{2\}(5-10) \%$ & 0.1004 \\
$\left\langle p_{T}\right\rangle\left(K^{-}\right)(20-30) \%$ & 0.0157 \\
$\left\langle p_{T}\right\rangle\left(K^{-}\right)(10-20) \%$ & 0.0152 \\
$\left\langle p_{T}\right\rangle\left(K^{-}\right)(5-10) \%$ & 0.0151 \\
\hline \hline
\end{tabular}

TABLE $X$. Seven observables at $\sqrt{s_{N N}}=62.4 \mathrm{GeV}$ with most weight on principal component 0 ( $82.42 \%$ of total variance).

\begin{tabular}{ll}
\hline \hline Observable & Weight \\
\hline$N(\Omega)(0-20) \%$ & 0.1511 \\
$N_{\text {ch }}(30-40) \%$ & 0.1338 \\
$d N_{\text {ch }} / d \eta$ at $\eta=0.1(20-25) \%$ & 0.1231 \\
$N_{\text {ch }}(20-30) \%$ & 0.1205 \\
$N_{\text {ch }}(10-20) \%$ & 0.1051 \\
$N_{\text {ch }}(5-10) \%$ & 0.0930 \\
$N_{\text {ch }}(0-5) \%$ & 0.0870 \\
\hline \hline
\end{tabular}

TABLE XI. Seven observables at $\sqrt{s_{N N}}=62.4 \mathrm{GeV}$ with most weight on principal component 1 (10.4\% of total variance).

\begin{tabular}{ll}
\hline \hline Observable & Weight \\
\hline$N(\Omega)(0-20) \%$ & 0.2199 \\
$v_{2}\{\mathrm{EP}\}(30-40) \%$ & 0.1323 \\
$v_{2}\{\mathrm{EP}\}(10-40) \%$ & 0.1313 \\
$v_{2}\{\mathrm{EP}\}(20-30) \%$ & 0.1077 \\
$v_{2}\{\mathrm{EP}\}(10-20) \%$ & 0.0806 \\
$\left\langle p_{T}\right\rangle(\Omega)(0-20) \%$ & 0.0548 \\
$\left\langle p_{T}\right\rangle\left(\pi^{-}\right)(20-30) \%$ & 0.0314 \\
\hline \hline
\end{tabular}

TABLE XII. Seven observables at $\sqrt{s_{N N}}=62.4 \mathrm{GeV}$ with most weight on principal component 2 (3.86\% of total variance).

\begin{tabular}{ll}
\hline \hline Observable & Weight \\
\hline$N(\Omega)(0-20) \%$ & 0.2674 \\
$v_{2}\{\mathrm{EP}\}(10-40) \%$ & 0.1463 \\
$v_{2}\{\mathrm{EP}\}(30-40) \%$ & 0.1412 \\
$v_{2}\{\mathrm{EP}\}(20-30) \%$ & 0.1123 \\
$v_{2}\{\mathrm{EP}\}(10-20) \%$ & 0.0819 \\
$\left\langle p_{T}\right\rangle(\Omega)(0-20) \%$ & 0.0243 \\
$R_{\text {long }}(0-5) \%$ & 0.0207 \\
\hline \hline
\end{tabular}


of $v_{2}\{\mathrm{EP}\}$ for charged particles within $|\eta|<0.3$ against the experimental value at $\eta=0.1$.

We have used the event plane $v_{2}$ also for comparisons with $v_{2}$ \{corr $\}$, which experimentally is determined with the correlation function method [38]. However, the preliminary data for $v_{2}$ \{corr $\}$ at $\sqrt{s_{N N}}=19.6 \mathrm{GeV}$ and $39 \mathrm{GeV}$ suggest that the magnitude of $v_{2}$ obtained by these two methods should be comparable in the investigated centrality range.

\section{APPENDIX B: PRINCIPAL COMPONENTS}

The number of principal components used is seven for each energy. This set of PCs explains over $99 \%$ of the total variance in each case. In Tables IV-XII, we list the most important observables for the first three principal components for each collision energy, as these already cover over $95 \%$ of the total variance.
[1] Y. Aoki, G. Endrodi, Z. Fodor, S. D. Katz, and K. K. Szabo, Nature (London) 443, 675 (2006)

[2] G. Endrodi, Z. Fodor, S. D. Katz, and K. K. Szabo, J. High Energy Phys. 04 (2011) 001.

[3] L. P. Csernai, J. I. Kapusta, and L. D. McLerran, Phys. Rev. Lett. 97, 152303 (2006).

[4] H. Niemi, K. J. Eskola, and R. Paatelainen, Phys. Rev. C 93, 024907 (2016).

[5] G. Denicol, A. Monnai, and B. Schenke, Phys. Rev. Lett. 116, 212301 (2016).

[6] J. E. Bernhard, J. S. Moreland, S. A. Bass, J. Liu, and U. Heinz, Phys. Rev. C 94, 024907 (2016).

[7] S. Plumari, G. L. Guardo, F. Scardina, and V. Greco, Nucl. Part. Phys. Proc. 276, 165 (2016).

[8] I. A. Karpenko, P. Huovinen, H. Petersen, and M. Bleicher, Phys. Rev. C 91, 064901 (2015).

[9] N. Demir and S. A. Bass, Phys. Rev. Lett. 102, 172302 (2009).

[10] G. S. Denicol, C. Gale, S. Jeon, and J. Noronha, Phys. Rev. C 88, 064901 (2013).

[11] L. Adamczyk, J. K. Adkins, G. Agakishiev, M. M. Aggarwal, Z. Ahammed, I. Alekseev, J. Alford, C. D. Anson, A. Aparin, D. Arkhipkin et al. (STAR Collaboration), Phys. Rev. C 92, 014904 (2015).

[12] L. Adamczyk, J. K. Adkins, G. Agakishiev, M. M. Aggarwal, Z. Ahammed, N. N. Ajitanand, I. Alekseev, D. M. Anderson, R. Aoyama, A. Aparin et al. (STAR Collaboration), Phys. Rev. C 96, 044904 (2017).

[13] S. A. Bass, M. Belkacem, M. Bleicher, M. Brandstetter, L. Bravina, C. Ernst, L. Gerland, M. Hofmann, S. Hofmann, J. Konopka et al., Prog. Part. Nucl. Phys. 41, 255 (1998).

[14] M. Bleicher, E. Zabrodin, C. Spieles, S. A. Bass, C. Ernst, S. Soff, L. Bravina, M. Belkacem, H. Weber, H. Stöcker, and W. Greiner, J. Phys. G 25, 1859 (1999).

[15] E. L. Bratkovskaya, M. Bleicher, M. Reiter, S. Soff, H. Stocker, M. van Leeuwen, S. A. Bass, and W. Cassing, Phys. Rev. C 69, 054907 (2004).

[16] H. Petersen, M. Bleicher, S. A. Bass, and H. Stocker, arXiv:0805.0567 [hep-ph].

[17] I. Karpenko, P. Huovinen, and M. Bleicher, Comput. Phys. Commun. 185, 3016 (2014).

[18] J. Steinheimer, S. Schramm, and H. Stocker, J. Phys. G 38, 035001 (2011).

[19] P. Huovinen and H. Petersen, Eur. Phys. J. A 48, 171 (2012).

[20] J. Novak, K. Novak, S. Pratt, J. Vredevoogd, C. E. ColemanSmith, and R. L. Wolpert, Phys. Rev. C 89, 034917 (2014).

[21] J. E. Bernhard, P. W. Marcy, C. E. Coleman-Smith, S. Huzurbazar, R. L. Wolpert, and S. A. Bass, Phys. Rev. C 91, 054910 (2015).
[22] J. Auvinen, K. Redlich, and S. A. Bass, J. Phys. Conf. Ser. 779, 012045 (2017).

[23] J. Auvinen, J. E. Bernhard, and S. A. Bass, Acta Phys. Polon. Supp. 10, 455 (2017).

[24] J. Auvinen, I. Karpenko, J. E. Bernhard, and S. A. Bass, Nucl. Phys. A 967, 784 (2017).

[25] D. Foreman-Mackey, D. W. Hogg, D. Lang, and J. Goodman, Publ. Astron. Soc. Pac. 125, 306 (2013).

[26] G. E. P. Box and D. R. Cox, J. Royal Stat. Soc. B 26, 211 (1964).

[27] B. B. Back, M. D. Baker, D. S. Barton, R. R. Betts, M. Ballintijn, A. A. Bickley, R. Bindel, A. Budzanowski, W. Busza, A. Carroll et al., Phys. Rev. Lett. 91, 052303 (2003).

[28] B. Alver, B. B. Back, M. D. Baker, M. Ballintijn, D. S. Barton, R. R. Betts, A. A. Bickley, R. Bindel, A. Budzanowski, W. Busza et al. (PHOBOS Collaboration), Phys. Rev. C 83, 024913 (2011).

[29] S. Das, Identified Particle Production and Freeze-out Dynamics in STAR at RHIC Beam Energy Scan Program, Ph.D. thesis, Institute of Physics. Bhubaneswar, India, 2015.

[30] B. I. Abelev, M. M. Aggarwal, Z. Ahammed, B. D. Anderson, D. Arkhipkin, G. S. Averichev, Y. Bai, J. Balewski, O. Barannikova, L. S. Barnby et al. (STAR Collaboration), Phys. Rev. C 79, 034909 (2009).

[31] M. A. Lisa, S. Pratt, R. Soltz, and U. Wiedemann, Annu. Rev. Nucl. Part. Sci. 55, 357 (2005).

[32] L. Adamczyk, G. Agakishiev, M. M. Aggarwal, Z. Ahammed, A. V. Alakhverdyants, I. Alekseev, J. Alford, B. D. Anderson, C. D. Anson, D. Arkhipkin et al. (STAR Collaboration), Phys. Rev. C 86, 054908 (2012).

[33] L. Adamczyk, J. K. Adkins, G. Agakishiev, M. M. Aggarwal, Z. Ahammed, I. Alekseev, A. Aparin, D. Arkhipkin, E. C. Aschenauer, A. Attri et al. (STAR Collaboration), Phys. Rev. C 93, 021903 (2016).

[34] M. Herrmann and G. F. Bertsch, Phys. Rev. C 51, 328 (1995).

[35] D. Teaney, Phys. Rev. C 68, 034913 (2003).

[36] B. B. Back, M. D. Baker, M. Ballintijn, D. S. Barton, R. R. Betts, A. A. Bickley, R. Bindel, W. Busza, A. Carroll, Z. Chai et al. (PHOBOS Collaboration), Phys. Rev. C 75, 024910 (2007).

[37] M. M. Aggarwal, Z. Ahammed, A. V. Alakhverdyants, I. Alekseev, J. Alford, B. D. Anderson, C. D. Anson, D. Arkhipkin, G. S. Averichev, J. Balewski et al. (STAR Collaboration), Phys. Rev. C 83, 024901 (2011).

[38] N. Magdy (STAR Collaboration), J. Phys. Conf. Ser. 779, 012060 (2017); (private communication).

[39] S. Chatterjee, S. Das, L. Kumar, D. Mishra, B. Mohanty, R. Sahoo, and N. Sharma, Adv. High Energy Phys. 2015, 349013 (2015) 